\title{
Optimization and validation of a QuEChERS-based method for the simultaneous environmental monitoring of 218 pesticide residues in clay loam soil
}

\author{
Andrea Acosta-Dacal ${ }^{\mathrm{a}, 1}$, Cristian Rial-Berriel ${ }^{\mathrm{a}, 1}$, Ricardo Díaz-Díaz ${ }^{\mathrm{b}}$, \\ María del Mar Bernal-Suárez ${ }^{\mathrm{b}}$, Octavio P. Luzardo ${ }^{\mathrm{a}, *}$ \\ a Toxicology Unit, Research Institute of Biomedical and Health Sciences (IUIBS), Universidad de Las Palmas de Gran Canaria, Paseo Blas Cabrera s/n, 35016 Las Palmas de Gran Canaria, Spain \\ b Department of Environmental Analysis, Technological Institute of the Canary Islands, C/Los Cactus no 68 35118, Polígono Industrial de Arinaga, Agüimes, Las Palmas, Canary Islands, Spain
}

\section{H I G H L I G H T S}

- Easy method for the sensitive quantification of 218 pesticides in clay loam soils

- One-step QuEChERS-based method without clean-up

- Tested in 18 agricultural soils in which 39 different residues were detected.

- Additional tested in soil samples from a wildlife poisoning incident

\section{A R T I C L E I N F O}

\section{Article history:}

Received 12 June 2020

Received in revised form 24 August 2020

Accepted 25 August 2020

Available online 26 August 2020

Editor: Jay Gan

\section{Keywords:}

Pesticides

Soils

Multi-residue extraction method

QuEChERS

LC-MS/MS

GC-MS/MS
G R A P H I C A L A B S T R A C T

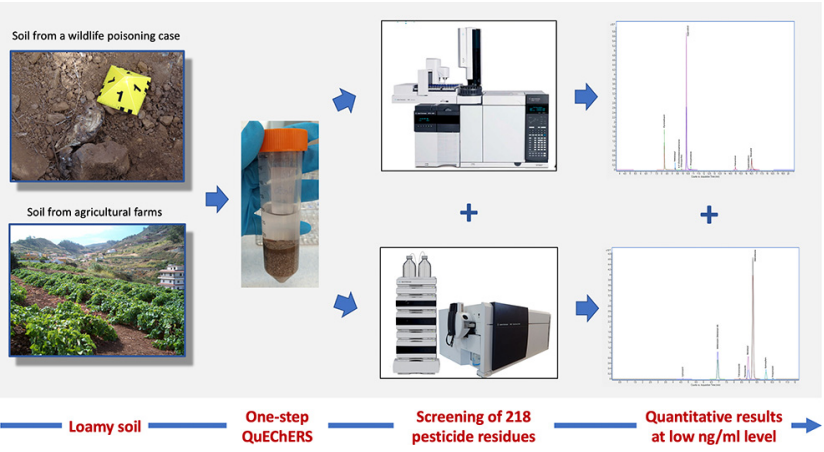

\begin{abstract}
A B S T R A C T
A modified QuEChERS method was optimized, validated and verified for the extraction of 218 pesticide residues in agricultural soil samples. The 218 analytes are extracted using a single step, without clean-up, with matrixmatched calibration, and two complementary techniques: liquid and gas chromatography tandem triple quad mass spectrometry (LC-MS/MS and GC-MS/MS). Some of the parameters such as salts, acidity of the extraction solvent, sample moisture and some mechanical changes in the procedure were optimized to improve the overall performance for the target compounds and the soil matrix. The method was fully validated on a representative agricultural soil sample of the Canary Islands (clay loam soil) in terms of linearity, accuracy and precision. To avoid matrix effects, matrix-matched calibration curves $\left(R^{2} \geq 0.99\right)$ were used for all target analytes. $100 \%$ of the compounds can be quantified with limits of quantification (LOQ) lower than the limit typically used in soils ( $50 \mathrm{ng} \mathrm{g}^{-1}$ ), with $92 \%$ of compounds presenting a LOQ that is at least 10 times lower than that normally required. The limits of detection (LOD) ranged between 0.024 and $6.25 \mathrm{ng} \mathrm{g}^{-1}$. The validated method was applied to a series of actual samples of agricultural soil $(n=18)$. In addition, as a further verification of its potential, the results of the application of the method in the investigation of clay loam soil samples that were obtained from underneath wildlife carcasses in the context of an environmental forensic investigation are also presented.
\end{abstract}

(c) 2020 Elsevier B.V. All rights reserved.

\footnotetext{
* Corresponding author at: Toxicology Unit, Clinical Sciences Department, Universidad de Las Palmas de Gran Canaria, Paseo Blas Cabrera Felipe s/n, 35016 Las Palmas, Spain.

E-mail address: octavio.perez@ulpgc.es (O.P. Luzardo).

${ }^{1}$ These authors contributed equally to the work, and therefore should be considered indistinctly as first authors.
} 


\section{Introduction}

The current use of pesticides in agriculture exceeds 350,000 tons per year. Their use is so common that the type of agriculture that uses them is called conventional agriculture, as opposed to other "alternative" that does not employ them. The continuous use of pesticides undoubtedly introduces unintended negative environmental impacts, such as the contamination of water sources and soils (Wang et al., 2019). When these agrochemicals are applied to the crop they usually end up in the soil from aerial and ground application (Kumari et al., 2008), where they can be retained by soil materials, be biologically or chemically degraded or be transported to other environmental compartments (Silva et al., 2019). Soil contamination by pesticides may affect soil functions, soil biodiversity, and the food security of subsequent crops (Wang et al., 2019). The high soil persistence of some pesticides is one of the most significant problems faced by farmers when moving to organic farming (Fenoll et al., 2009). However, there is no European regulation that obliges to monitoring programmes at the supranational level, and therefore there is no harmonised analytical methodology and no official list of residues to be monitored in soil. Probably for this reason, there is not clear overview of the level of contamination of agricultural soils at the international level. Most of the available studies focus on the monitoring of a discrete number of permitted (Han et al., 2017; Karasali et al., 2016; Kosubova et al., 2020; Suszter and Ambrus, 2017; Vasickova et al., 2019), or prohibited pesticides (Barron et al., 2017; Eudoxie et al., 2019; Hwang et al., 2018; Kosubova et al., 2020). One of the most recent and complete studies found that $83 \%$ of the samples were contaminated with one or more pesticides (Silva et al., 2019). In the case of Spain, a multiannual programme for official control of agricultural production (2016-2020) is currently in force, which focuses mainly on research on pesticide residues in plants, but it also includes soil and water analysis. However, the programme does not refer to the methodology to be used in the analysis of soil samples.

Given the above, there is an increasing need for validated methods capable of quantifying pesticide residues in soil, similar to those used for the mandatory determination of pesticide residues in vegetables (EC, 2019a) or in water (EC, 2000). The extraction procedure employed has to be capable of extracting both the bound and the non-bound residues, with minimal co-extraction of the matrix constituents (Otalvaro and Brigante, 2018).

Pesticide extraction in soils has been carried out using many different extraction techniques: liquid-solid extraction (LSE) (Djurovic et al., 2012), ultrasonic solvent extraction (Castro et al., 2001; Tor et al., 2006), Soxhlet extraction (Wong et al., 2010; Zhou et al., 2013), microwaved-assisted extraction (de Andrea et al., 2001; Merdassa et al., 2013), pressurized liquid extraction (Masia et al., 2015; Vidal et al., 2010), supercritical fluid extraction (Forero-Mendieta et al., 2012; Snyder et al., 1994), solid-phase microextraction (Djurovic et al., 2010; Fernandez-Alvarez et al., 2008), and most recently, modified QuEChERS (Asensio-Ramos et al., 2010; Lesueur et al., 2008), which seems to be one of the most convenient due to the high extraction yields that can be achieved. (Perestrelo et al., 2019). Subsequent analyses have been mostly performed by gas chromatography (GC) (Pastor-Belda et al., 2015; Redondo et al., 1996; Salemi et al., 2013; Zhao et al., 2018), and liquid chromatography (LC) usually tandem coupled to mass spectrometry (LC-MS/MS) (Silva et al., 2019; Zhang et al., 2011).

The aim of this work was to optimize and validate a modified QuEChERS-based extraction method for the quantitative determination of multiple pesticide residues in clay loam soils, to include as many pesticides currently investigated in food products in the EU as possible, but also other environmentally relevant pesticides. The validated method allows the accurate quantification of 218 pesticides and metabolites by means of two complementary analyses by LC-MS/MS and GC-MS/MS. The method was fully applied to a series of actual samples of agricultural soil. In addition, the results of its application to a clay loam soil sample obtained from underneath wildlife carcasses in the context of an environmental forensic investigation are presented.

\section{Materials and methods}

\subsection{Reagents and chemicals}

Standard stock solutions of the pesticides subjected to official control in agricultural commodities (EC, 2019a) were purchased from CPA Chem (Stara Zagora, Bulgaria) in 10 compatible mixes $\left(10 \mu \mathrm{g} \mathrm{mL}{ }^{-1}\right.$ each). The rest of the pesticides and the isotopically labeled Procedural Internal Standards (P-IS) were purchased from Dr. Ehrenstorfer GmbH (Augsburg, Germany) and Sigma-Aldrich (Büchs, Switzerland), all with a purity superior to $97.1 \%$.

LC-MS grade formic acid (FA, $\mathrm{HCOOH})$, acetic acid (AA, $\left.\mathrm{CH}_{3} \mathrm{COOH}\right)$, acetonitrile $(\mathrm{ACN})$ and methanol $(\mathrm{MeOH})$ were purchased from Honeywell (Morristown, NJ). Ultrapure water was produced in the laboratory using a Gradient A10 Milli-Q System (Millipore, Bedfore, MA, USA). AOAC and EN method QuEChERS salts, and Agilent d-SPE Enhanced Matrix Removal-Lipid (EMR-lipid) were acquired from Agilent Technologies (Palo Alto, USA). Ammonium acetate $\left(\mathrm{NH}_{4} \mathrm{CH}_{3} \mathrm{CO}_{2}\right)$ and ammonium formate $\left(\mathrm{NH}_{4} \mathrm{CO}_{2}\right)$ were obtained from Fisher Scientific (Loughborough, UK).

\subsection{Standard stock solutions and mixes}

For those pesticides not included in the multiannual coordinated programme (EC, 2019a; Ruiz-Suarez et al., 2015) individual standard stock solutions at a concentration of $1000 \mu \mathrm{g} \mathrm{mL}{ }^{-1}$ were prepared. Intermediate solutions ( $1 \mu \mathrm{g} \mathrm{mL} \mathrm{L}^{-1}$ ) were used to optimize the mass spectrometer conditions. A working solution containing all the analytes at a final concentration of $0.833 \mu \mathrm{g} \mathrm{mL}^{-1}$ /each was prepared. Additionally, A P-IS mix was prepared at a concentration of $1 \mu \mathrm{g} \mathrm{mL}^{-1}$ in acetonitrile. Matrix-matched calibration curves were prepared with the standard working mix solution, either in soil matrix extracted following the recommended procedure for GC-MS/MS or in a mixture of this extract and ultrapure water $(1: 1, v / v)$ for LC-MS/MS. All 218 compounds and P-ISs finally included are listed in Table 1. Working solutions were checked periodically for stability.

\subsection{Sample selection and pre-treatment}

For the method development we chose soil samples from two farms dedicated to organic production. On the basis of its physicochemical properties, this composite can be classified as clay loam soil: $\mathrm{pH} 4.88$, electrical conductivity $209 \mu \mathrm{S} \mathrm{cm}{ }^{-1}$, oxidizable organic carbon $2.19 \%$ (which is equivalent to approx. 3.9\% organic matter), 6\% moisture and particle size distribution: $29.5 \%$ clay, $28.3 \%$ fine silt, $11.3 \%$ thick slit, $11.5 \%$ coarse sand, and $19.4 \%$ fine sand.

Additionally, to verify the applicability of the method after the validation process, the method was applied to a series of 18 clay loam agricultural soil samples from the Canary Islands, Spain). In each sampling plot, a composite sample was prepared from at least four subsamples collected at depths between 20 and $30 \mathrm{~cm}$. Then, the soil was homogenized, air-dried at room temperature and sieved ( $2 \mathrm{~mm}$ mesh). Additionally, two clay loam soil samples from a site where animal remains were found in the context of a malicious wildlife poisoning episode were also analyzed.

For the classification of soil samples, electrical conductivity and $\mathrm{pH}$ were measured with suitable electrodes in soil-water suspensions $(1: 5, w / v)$. Moisture was calculated as the difference between the air-dried soil weight and the weight after $24 \mathrm{~h}$ in an oven at $105{ }^{\circ} \mathrm{C}$. Particle distribution was obtained with the hydrometer method (Ashworth et al., 2001). The oxidizable organic carbon to calculate the organic matter content was determined according to the spectrophotometric method in which the absorbance reading is 
Table 1

Compounds analyzed in soil with the category of use, legal status, analysis technique and mass spectrometric conditions.

\begin{tabular}{|c|c|c|c|c|c|c|c|c|c|c|c|c|c|}
\hline \multirow[t]{2}{*}{$\mathrm{N}^{\circ}$} & \multirow[t]{2}{*}{ Compound } & \multirow[t]{2}{*}{ Category $^{\mathrm{a}}$} & \multirow{2}{*}{$\begin{array}{l}\text { Legal status in } \\
\text { the } \mathrm{EU}^{\mathrm{b}}\end{array}$} & \multirow{2}{*}{$\begin{array}{l}\text { Subjected to } \\
\text { MRL }^{c}\end{array}$} & \multirow[t]{2}{*}{ Technique $^{\mathrm{d}}$} & \multirow[t]{2}{*}{ Retention time (min) } & \multirow{2}{*}{$\begin{array}{c}\mathrm{LOQ} \\
\mathrm{ng} \mathrm{g}^{-1}\end{array}$} & \multirow[t]{2}{*}{ Polarity } & \multicolumn{2}{|c|}{ Quantification } & \multicolumn{2}{|c|}{ Confirmation } & \multirow[t]{2}{*}{ Fragmentor } \\
\hline & & & & & & & & & $\begin{array}{l}\text { MRM transition } \\
(\mathrm{m} / \mathrm{z})\end{array}$ & $\begin{array}{c}\mathrm{CE} \\
(\mathrm{eV})\end{array}$ & $\begin{array}{l}\text { MRM transition } \\
(\mathrm{m} / \mathrm{z})\end{array}$ & $\begin{array}{c}\mathrm{CE} \\
(\mathrm{eV})\end{array}$ & \\
\hline 1 & 4.4'-Dichlorobenzophenone (metabolite of dicofol) & Met & - & No & GC & 9.97 & 0.5 & Positive & $250.0 \rightarrow 139.0$ & 15 & $250.0 \rightarrow 215.0$ & 5 & 70 \\
\hline 2 & 4.4'-Dicofol & POP & Not approved & Yes & GC & 13.14 & 20.0 & Positive & $251.0 \rightarrow 111.0$ & 15 & $251.0 \rightarrow 139.0$ & 45 & 70 \\
\hline 3 & Abamectine & $\mathrm{I}, \mathrm{A}, \mathrm{AH}$ & Approved & Yes & LC & 10.93 & 20.0 & Positive & $890.5 \rightarrow 567.1$ & 50 & $895.5 \rightarrow 751.4$ & 45 & 160 \\
\hline 4 & Acephate & I & Not approved & Yes & LC & 1.90 & 0.5 & Positive & $184.0 \rightarrow 143.0$ & 0 & $184.0 \rightarrow 95.0$ & 20 & 70 \\
\hline 5 & Acetamiprid & I & Approved & Yes & LC & 4.39 & 1.0 & Positive & $223.1 \rightarrow 126.0$ & 45 & $223.1 \rightarrow 90.0$ & 27 & 140 \\
\hline 6 & Acrinathrin & $\mathrm{I}, \mathrm{A}$ & Approved & Yes & $\mathrm{LC}$ & 10.65 & 5.0 & Positive & $559.0 \rightarrow 208.0$ & 30 & $559.0 \rightarrow 181.0$ & 10 & 76 \\
\hline 7 & Aldicarb & I & Not approved & Yes & LC & 5.09 & 0.5 & Positive & $116.0 \rightarrow 89.1$ & 4 & $208.0 \rightarrow 116.0$ & 0 & 100 \\
\hline 8 & Aldicarb sulfone & Met & - & Yes & LC & 3.15 & 1.0 & Positive & $240.1 \rightarrow 76.0$ & 13 & $223.1 \rightarrow 86.1$ & 13 & 120 \\
\hline 9 & Atrazine & $\mathrm{H}$ & Not approved & No & LC & 6.70 & 1.0 & Positive & $216.0 \rightarrow 68.1$ & 15 & $216.0 \rightarrow 103.8$ & 30 & 130 \\
\hline 10 & Azinphos methyl & I & Not approved & Yes & LC & 7.22 & 0.5 & Positive & $318.0 \rightarrow 132.1$ & 15 & $318.0 \rightarrow 261.0$ & 8 & 90 \\
\hline 11 & Azoxystrobin & $\mathrm{F}$ & Approved & Yes & LC & 7.53 & 0.5 & Positive & $404.1 \rightarrow 344.1$ & 8 & $404.1 \rightarrow 329.1$ & 32 & 110 \\
\hline 12 & Benalaxyl & $\mathrm{F}$ & Approved & No & LC & 8.90 & 0.5 & Positive & $326.2 \rightarrow 148.0$ & 20 & $326.2 \rightarrow 208.0$ & 12 & 90 \\
\hline 13 & Bendiocarb & I & Not approved & No & $\mathrm{LC}$ & 5.84 & 1.0 & Positive & $224.1 \rightarrow 166.9$ & 8 & $224.1 \rightarrow 108.9$ & 30 & 100 \\
\hline 14 & Bifenthrin & I & Not approved & Yes & GC & 13.84 & 20.0 & Positive & $181.2 \rightarrow 165.2$ & 25 & $181.2 \rightarrow 115.0$ & 60 & 70 \\
\hline 15 & Bitertanol & $\mathrm{F}$ & Not approved & Yes & LC & 9.17 & 2.5 & Positive & $338.2 \rightarrow 70.0$ & 5 & $338.2 \rightarrow 269.2$ & 4 & 100 \\
\hline 16 & Boscalid (formely nicobifen) & $\mathrm{F}$ & Approved & Yes & GC & 16.53 & 5.0 & Positive & $342.0 \rightarrow 140.0$ & 15 & $342.0 \rightarrow 112.0$ & 45 & 70 \\
\hline 17 & Bromopropylate & A & Not approved & Yes & GC & 13,84 & 20.0 & Positive & $341,0 \rightarrow 183,0$ & 15 & $341,0 \rightarrow 157,0$ & 45 & 70 \\
\hline 18 & Bromuconazole (two isomers) & $\mathrm{F}$ & Approved & No & LC & $8.09 / 8.67$ & 2.5 & Positive & $378.0 \rightarrow 159.0$ & 35 & $376.0 \rightarrow 159.0$ & 32 & 150 \\
\hline 19 & Bupirimate & $\mathrm{F}$ & Approved & Yes & LC & 8.30 & 0.5 & Positive & $317.2 \rightarrow 108.1$ & 28 & $317.2 \rightarrow 166.1$ & 18 & 100 \\
\hline 20 & Buprofezin & I & Approved & Yes & LC & 9.79 & 0.5 & Positive & $306.1 \rightarrow 201.0$ & 12 & $306.1 \rightarrow 116.0$ & 12 & 140 \\
\hline 21 & Cadusafos (ebufos) & $\mathrm{I}, \mathrm{AH}$ & Not approved & No & LC & 9.33 & 0.5 & Positive & $271.1 \rightarrow 159.0$ & 22 & $271.1 \rightarrow 131.0$ & 16 & 100 \\
\hline 22 & Carbaryl & I & Not approved & Yes & LC & 6.16 & 0.5 & Positive & $202.1 \rightarrow 145.1$ & 4 & $202.1 \rightarrow 127.1$ & 28 & 90 \\
\hline 23 & Carbofuran & $\mathrm{I}, \mathrm{AH}$ & Not approved & Yes & LC & 5.88 & 0.5 & Positive & $222.1 \rightarrow 123.1$ & 20 & $222.1 \rightarrow 165.1$ & 30 & 80 \\
\hline 24 & Carbofuran-3-hydroxy & Met & - & Yes & $\mathrm{LC}$ & 4.37 & 0.5 & Positive & $238.1 \rightarrow 163.1$ & 10 & $238.1 \rightarrow 181.1$ & 10 & 110 \\
\hline 25 & Chlorantraniliprole & I & Approved & Yes & LC & 7.27 & 1.0 & Positive & $483.9 \rightarrow 452.9$ & 16 & $483.9 \rightarrow 285.9$ & 8 & 105 \\
\hline 26 & Chlorfenapyr & I, A & Not approved & Yes & GC & 11.97 & 10.0 & Positive & $247.0 \rightarrow 227.0$ & 15 & $328.0 \rightarrow 247.0$ & 20 & 70 \\
\hline 27 & Chlorfenvinphos & I & Not approved & No & LC & 8.98 & 1.0 & Positive & $358.9 \rightarrow 155.1$ & 8 & $361.1 \rightarrow 154.9$ & 34 & 105 \\
\hline 28 & Chlorobenzilate & A & Not approved & No & GC & 12.09 & 10.0 & Positive & $251.0 \rightarrow 139.0$ & 15 & $251.0 \rightarrow 111.0$ & 40 & 70 \\
\hline 29 & Chlorpropham & $\mathrm{H}$ & Not approved & Yes & GC & 7.05 & 0.5 & Positive & $213.0 \rightarrow 127.0$ & 15 & $153.0 \rightarrow 90.0$ & 10 & 70 \\
\hline 30 & Chlorpyrifos & I & Not approved & Yes & GC & 9.88 & 5.0 & Positive & $314.0 \rightarrow 258.0$ & 15 & $314.0 \rightarrow 286.0$ & 5 & 70 \\
\hline 31 & Chlorpyrifos methyl & I & Not approved & Yes & GC & 9.07 & 5.0 & Positive & $286.0 \rightarrow 93.0$ & 25 & $286.0 \rightarrow 271.0$ & 15 & 70 \\
\hline 32 & Chlorthal dimethyl & $\mathrm{H}$ & Not approved & No & GC & 9.98 & 2.5 & Positive & $300.9 \rightarrow 166.9$ & 55 & $300.9 \rightarrow 222.9$ & 25 & 70 \\
\hline 33 & Clofentezine & A & Approved & Yes & LC & 9.12 & 2.5 & Positive & $303.1 \rightarrow 138.0$ & 12 & $303.1 \rightarrow 102.0$ & 40 & 120 \\
\hline 34 & Clothianidin & I & Not approved & Yes & LC & 4.09 & 2.5 & Positive & $250.0 \rightarrow 169.0$ & 8 & $250.0 \rightarrow 131.9$ & 8 & 100 \\
\hline 35 & Coumachlor & $\mathrm{R}$ & Not approved & No & LC & 8.55 & 0.5 & Positive & $343.1 \rightarrow 162.8$ & 15 & $343.1 \rightarrow 285.0$ & 15 & 120 \\
\hline 36 & Coumaphos & I, A & Not approved & No & LC & 8.92 & 1.0 & Positive & $363.0 \rightarrow 227.0$ & 30 & $363.0 \rightarrow 306.9$ & 15 & 120 \\
\hline 37 & Cyazofamid & $\mathrm{F}$ & Approved & Yes & $\mathrm{LC}$ & 8.42 & 5.0 & Positive & $325.0 \rightarrow 108.0$ & 20 & $325.0 \rightarrow 261.1$ & 15 & 90 \\
\hline 38 & Cyflufenamid & $\mathrm{F}$ & Approved & Yes & $\mathrm{LC}$ & 9.12 & 2.5 & Positive & $413.1 \rightarrow 223.1$ & 23 & $413.1 \rightarrow 295.1$ & 33 & 70 \\
\hline 39 & Cyfluthrin (sum of four isomers) & I & Not approved $^{\mathrm{e}}$ & Yes & GC & $16.11 / 16.2 / 16.27 / 16.31$ & 10.0 & Positive & $226.0 \rightarrow 206.0$ & 25 & $226.0 \rightarrow 199.0$ & 10 & 70 \\
\hline 40 & Cyhalothrin (lambda isomer) & I & Approved & Yes & LC & 10.43 & 20.0 & Positive & $467.0 \rightarrow 225.0$ & 10 & $467.0 \rightarrow 141.0$ & 46 & 66 \\
\hline 41 & Cymoxanil & $\mathrm{F}$ & Approved & Yes & LC & 4.64 & 0.5 & Positive & $199.1 \rightarrow 128.0$ & 4 & $199.1 \rightarrow 110.9$ & 12 & 90 \\
\hline 42 & Cypermethrin (sum of four isomers) & I & Approved ${ }^{\mathrm{f}}$ & Yes & GC & $16.42 / 16.51 / 16.60 / 16.62$ & 5.0 & Positive & $163.0 \rightarrow 127.0$ & 15 & $163.0 \rightarrow 109.0$ & 5 & 70 \\
\hline 43 & Cyproconazole (two isomers) & $\mathrm{F}$ & Approved & Yes & LC & $7.97 / 8.18$ & 0.5 & Positive & $292.2 \rightarrow 70.2$ & 18 & $292.2 \rightarrow 125.1$ & 24 & 100 \\
\hline 44 & Cyprodinil & $\mathrm{F}$ & Approved & Yes & GC & 10.33 & 2.5 & Positive & $224.0 \rightarrow 118.0$ & 45 & $224.0 \rightarrow 104.0$ & 25 & 70 \\
\hline 45 & Deltamethrin & I, A & Approved & Yes & GC & 18.06 & 5.0 & Positive & $181.0 \rightarrow 152.1$ & 15 & $251.0 \rightarrow 172.0$ & 25 & 70 \\
\hline 46 & Demeton-S-methyl & I, A & Not approved & No & LC & 5.92 & 0.5 & Positive & $230.9 \rightarrow 88.9$ & 5 & $230.9 \rightarrow 61.0$ & 30 & 50 \\
\hline 47 & Demeton-S-methyl-sulfone (Dioxydemeton) & I, A & Not approved & No & LC & 3.54 & 0.5 & Positive & $263.0 \rightarrow 169.0$ & 24 & $263.0 \rightarrow 109.0$ & 12 & 120 \\
\hline 48 & Diazinon & I & Not approved & Yes & GC & 8.21 & 0.5 & Positive & $304.0 \rightarrow 179.0$ & 15 & $137.1 \rightarrow 84.0$ & 20 & 70 \\
\hline 49 & Dichlofluanid & $\mathrm{F}$ & Not approved & No & GC & 9.68 & 2.5 & Positive & $224.0 \rightarrow 123.0$ & 10 & $226.0 \rightarrow 123.0$ & 15 & 70 \\
\hline 50 & Dichloran & F, MB, WP & Not approved & Yes & GC & 7.75 & 2.5 & Positive & $206.0 \rightarrow 176.0$ & 25 & $206.0 \rightarrow 148.0$ & 10 & 70 \\
\hline 51 & Diethathyl ethyl & $\mathrm{H}$ & Not approved & No & LC & 8.66 & 0.5 & Positive & $312.2 \rightarrow 238.1$ & 15 & $312.2 \rightarrow 162.0$ & 30 & 120 \\
\hline 52 & Diethofencarb & F, MB, WP & Approved & Yes & LC & 7.52 & 20.0 & Positive & $268.2 \rightarrow 226.1$ & 5 & $268.2 \rightarrow 152.0$ & 20 & 110 \\
\hline
\end{tabular}




\begin{tabular}{|c|c|c|c|c|c|c|c|c|c|c|c|c|c|}
\hline \multirow[t]{2}{*}{$\mathrm{N}^{\circ}$} & \multirow[t]{2}{*}{ Compound } & \multirow[t]{2}{*}{ Category $^{a}$} & \multirow{2}{*}{$\begin{array}{l}\text { Legal status in } \\
\text { the } \mathrm{EU}^{\mathrm{b}}\end{array}$} & \multirow{2}{*}{$\begin{array}{l}\text { Subjected to } \\
\text { MRL }^{c}\end{array}$} & \multirow[t]{2}{*}{ Technique $^{\mathrm{d}}$} & \multirow[t]{2}{*}{ Retention time (min) } & \multirow{2}{*}{$\begin{array}{c}\mathrm{LOQ} \\
\mathrm{ng} \mathrm{g}^{-1}\end{array}$} & \multirow[t]{2}{*}{ Polarity } & \multicolumn{2}{|c|}{ Quantification } & \multicolumn{2}{|c|}{ Confirmation } & \multirow[t]{2}{*}{ Fragmentor } \\
\hline & & & & & & & & & $\begin{array}{l}\text { MRM transition } \\
(\mathrm{m} / \mathrm{z})\end{array}$ & $\begin{array}{c}\mathrm{CE} \\
(\mathrm{eV})\end{array}$ & $\begin{array}{l}\text { MRM transition } \\
(\mathrm{m} / \mathrm{z})\end{array}$ & $\begin{array}{l}\mathrm{CE} \\
(\mathrm{eV})\end{array}$ & \\
\hline 53 & Difenoconazole & F, MB, WP & Approved & Yes & LC & 9.35 & 1.0 & Positive & $406.1 \rightarrow 250.9$ & 28 & $406.1 \rightarrow 337.0$ & 16 & 176 \\
\hline 54 & Diflubenzuron & I & Approved & Yes & LC & 8.55 & 1.0 & Positive & $311.0 \rightarrow 158.0$ & 32 & $311.0 \rightarrow 141.0$ & 8 & 90 \\
\hline 55 & Diflufenican & $\mathrm{H}$ & Approved & No & LC & 9.44 & 0.5 & Positive & $395.1 \rightarrow 266.0$ & 24 & $395.1 \rightarrow 246.0$ & 36 & 150 \\
\hline 56 & Dimethenamide & $\mathrm{H}$ & Approved & No & LC & 7.65 & 0.5 & Positive & $276.1 \rightarrow 244.1$ & 10 & $276.1 \rightarrow 168.1$ & 20 & 125 \\
\hline 57 & Dimethoate & I & Not approved & Yes & LC & 4.32 & 0.5 & Positive & $230.0 \rightarrow 198.8$ & 16 & $230.0 \rightarrow 125.0$ & 0 & 70 \\
\hline 58 & Dimethomorph (two isomers) & F, MB, WP & Approved & Yes & $\mathrm{LC}$ & $7.68 / 7.94$ & 1.0 & Positive & $388.1 \rightarrow 301.1$ & 20 & $388.1 \rightarrow 165.1$ & 32 & 180 \\
\hline 59 & Diniconazole-M & F, MB, WP & Not approved & Yes & LC & 9.26 & 1.0 & Positive & $326.1 \rightarrow 70.0$ & 28 & $328.1 \rightarrow 70.0$ & 28 & 110 \\
\hline 60 & Dinocap & $\mathrm{F}, \mathrm{MB}, \mathrm{WP}$ & Not approved & No & LC & 10.45 & 20.0 & Negative & $295.4 \rightarrow 193.0$ & 30 & $295.4 \rightarrow 163.0$ & 40 & 150 \\
\hline 61 & Diphenylamine & PHP & Not approved & Yes & LC & 4.24 & 20.0 & Positive & $170.0 \rightarrow 65.0$ & 65 & $170.0 \rightarrow 93.0$ & 40 & 200 \\
\hline 62 & Endosulfan alfa & POP & - & Yes & GC & 11.18 & 0.5 & Positive & $241.0 \rightarrow 206.0$ & 10 & $195.0 \rightarrow 160.0$ & 15 & 70 \\
\hline 63 & Endosulfan beta & POP & - & Yes & GC & 12.19 & 5.0 & Positive & $195.0 \rightarrow 125.0$ & 25 & $195.0 \rightarrow 159.0$ & 10 & 70 \\
\hline 64 & EPN & $\mathrm{I}, \mathrm{A}$ & Not approved & No & LC & 9.38 & 10.0 & Positive & $324.0 \rightarrow 157.0$ & 25 & $324.0 \rightarrow 296.0$ & 14 & 88 \\
\hline 65 & Epoxiconazole & $\mathrm{F}$ & Approved & Yes & LC & 8.40 & 1.0 & Positive & $330.0 \rightarrow 100.9$ & 50 & $330.0 \rightarrow 120.9$ & 24 & 120 \\
\hline 66 & Esfenvalerate & I & Approved & No & GC & 17.54 & 5.0 & Positive & $167.0 \rightarrow 125.1$ & 45 & $225.0 \rightarrow 119.0$ & 15 & 70 \\
\hline 67 & Ethion (diethion) & $\mathrm{I}, \mathrm{A}$ & Not approved & Yes & LC & 9.95 & 0.5 & Positive & $385.0 \rightarrow 199.0$ & 5 & $385.0 \rightarrow 171.0$ & 10 & 100 \\
\hline 68 & Ethofumesate & $\mathrm{H}$ & Approved & No & GC & 9.54 & 5.0 & Positive & $286.0 \rightarrow 207.0$ & 5 & $286.0 \rightarrow 161.0$ & 20 & 70 \\
\hline 69 & Ethoprophos & $\mathrm{I}, \mathrm{AH}$ & Not approved & No & LC & 8.33 & 0.5 & Positive & $243.1 \rightarrow 97.0$ & 30 & $243.1 \rightarrow 130.9$ & 15 & 90 \\
\hline 70 & Etofenprox & $\mathrm{I}, \mathrm{A}$ & Approved & Yes & LC & 11.13 & 1.0 & Positive & $394.0 \rightarrow 359.0$ & 10 & $394.0 \rightarrow 135.1$ & 40 & 66 \\
\hline 71 & Etoxazole & A & Approved & Yes & LC & 10.27 & 0.5 & Positive & $360.1 \rightarrow 304.0$ & 28 & $360.1 \rightarrow 113.0$ & 26 & 160 \\
\hline 72 & Famoxadone & $\mathrm{H}$ & Approved & Yes & LC & 9.01 & 2.5 & Positive & $392.1 \rightarrow 330.9$ & 5 & $392.1 \rightarrow 238.1$ & 12 & 110 \\
\hline 73 & Fenamidone & $\mathrm{F}$ & Not approved & Yes & LC & 7.67 & 1.0 & Positive & $312.0 \rightarrow 92.2$ & 28 & $312.0 \rightarrow 236.1$ & 14 & 100 \\
\hline 74 & Fenamiphos & $\mathrm{I}, \mathrm{AH}$ & Approved & Yes & LC & 8.57 & 0.5 & Positive & $304.1 \rightarrow 217.1$ & 20 & $304.1 \rightarrow 202.0$ & 36 & 120 \\
\hline 75 & Fenamiphos sulfone & Met & - & Yes & LC & 6.19 & 0.5 & Positive & $336.1 \rightarrow 308.1$ & 23 & $336.1 \rightarrow 188.0$ & 31 & 120 \\
\hline 76 & Fenamiphos sulfoxide & Met & - & Yes & LC & 6.03 & 1.0 & Positive & $320.1 \rightarrow 233.0$ & 20 & $320.1 \rightarrow 108.1$ & 44 & 120 \\
\hline 77 & Fenarimol & F, MB, WP & Not approved & Yes & GC & 15.02 & 10.0 & Positive & $139.0 \rightarrow 75.0$ & 30 & $139.0 \rightarrow 111.0$ & 15 & 70 \\
\hline 78 & Fenazaquin & A & Approved & Yes & LC & 10.65 & 0.5 & Positive & $307.2 \rightarrow 161.1$ & 25 & $307.2 \rightarrow 131.0$ & 16 & 130 \\
\hline 79 & Fenbuconazole & $\mathrm{F}, \mathrm{V}$ & Approved & Yes & LC & 8.51 & 2.5 & Positive & $337.1 \rightarrow 70.0$ & 40 & $337.1 \rightarrow 125.1$ & 33 & 160 \\
\hline 80 & Fenbutatin oxide & $\mathrm{I}, \mathrm{A}$ & Not approved & Yes & $\mathrm{LC}$ & 11.51 & 2.5 & Positive & $519.0 \rightarrow 90.9$ & 65 & $519.0 \rightarrow 197.0$ & 55 & 180 \\
\hline 81 & Fenitrothion & I & Not approved & Yes & GC & 9.50 & 10.0 & Positive & $277.0 \rightarrow 109.0$ & 15 & $277.0 \rightarrow 260.0$ & 5 & 70 \\
\hline 82 & Fenoxycarb & I & Approved & Yes & LC & 8.63 & 0.5 & Positive & $302.1 \rightarrow 88.0$ & 10 & $302.1 \rightarrow 116.1$ & 20 & 110 \\
\hline 83 & Fenpropathrin & $\mathrm{I}, \mathrm{A}$ & Not approved & Yes & LC & 10.37 & 1.0 & Positive & $367.2 \rightarrow 125.0$ & 16 & $350.2 \rightarrow 125.0$ & 16 & 72 \\
\hline 84 & Fenpropimorph & $\mathrm{F}$ & Not approved & Yes & LC & 7.39 & 0.5 & Positive & $304.3 \rightarrow 147.1$ & 30 & $304.3 \rightarrow 130.0$ & 25 & 120 \\
\hline 85 & Fenpyroximate & A & Approved & Yes & LC & 10.42 & 0.5 & Positive & $422.2 \rightarrow 366.2$ & 12 & $422.2 \rightarrow 135.0$ & 36 & 160 \\
\hline 86 & Fenthion & $\mathrm{I}, \mathrm{A}$ & Not approved & Yes & LC & 8.82 & 2.5 & Positive & $279.0 \rightarrow 168.8$ & 8 & $279.0 \rightarrow 247.1$ & 18 & 98 \\
\hline 87 & Fenthion oxon & Met & - & Yes & LC & 7.26 & 0.5 & Positive & $263.1 \rightarrow 231.2$ & 16 & $263.1 \rightarrow 216.0$ & 24 & 120 \\
\hline 88 & Fenthion oxon sulfone & Met & - & Yes & LC & 4.61 & 0.5 & Positive & $295.0 \rightarrow 217.0$ & 15 & $295.0 \rightarrow 104.2$ & 20 & 110 \\
\hline 89 & Fenthion oxon sulfoxide & Met & - & Yes & LC & 4.46 & 0.5 & Positive & $279.0 \rightarrow 104.1$ & 20 & $279.0 \rightarrow 264.2$ & 28 & 110 \\
\hline 90 & Fenthion sulfone & Met & - & Yes & LC & 6.32 & 0.5 & Positive & $311.0 \rightarrow 125.0$ & 28 & $311.0 \rightarrow 109.0$ & 22 & 140 \\
\hline 91 & Fenthion sulfoxide & Met & - & Yes & LC & 6.10 & 0.5 & Positive & $295.0 \rightarrow 280.0$ & 15 & $295.0 \rightarrow 108.9$ & 30 & 140 \\
\hline 92 & Fenvalerate & I & Not approved & Yes & GC & 17.34 & 5.0 & Positive & $167.0 \rightarrow 125.1$ & 45 & $225.0 \rightarrow 119.0$ & 15 & 70 \\
\hline 93 & Fipronil & $\mathrm{I}, \mathrm{V}$ & Not approved & Yes & LC & 8.61 & 2.5 & Negative & $435.0 \rightarrow 330.0$ & 26 & $435.0 \rightarrow 249.9$ & 12 & 116 \\
\hline 94 & Fipronil sulfide & Met & - & Yes & GC & 10.44 & 5.0 & Positive & $351.0 \rightarrow 255.0$ & 20 & $420.0 \rightarrow 351.0$ & 35 & 70 \\
\hline 95 & Fluazinam & $\mathrm{F}$ & Approved & No & LC & 9.94 & 2.5 & Negative & $462.9 \rightarrow 416.0$ & 10 & $462.9 \rightarrow 398.0$ & 9 & 140 \\
\hline 96 & Flubendiamide & I & Approved & Yes & LC & 8.75 & 2.5 & Positive & $408.0 \rightarrow 274.0$ & 15 & $408.0 \rightarrow 256.0$ & 30 & 120 \\
\hline 97 & Flucythrinate (two isomers) & $\mathrm{I}, \mathrm{A}$ & Not approved & No & GC & $16.63 / 16.82$ & 5.0 & Positive & $199.1 \rightarrow 107.1$ & 25 & $156.9 \rightarrow 107.1$ & 15 & 70 \\
\hline 98 & Fludioxonil & $\mathrm{F}$ & Approved & Yes & LC & 7.71 & 5.0 & Negative & $247.0 \rightarrow 180.0$ & 62 & $247.0 \rightarrow 125.9$ & 32 & 152 \\
\hline 99 & Flufenoxuron & $\mathrm{I}, \mathrm{A}$ & Not approved & Yes & LC & 10.30 & 0.5 & Positive & $489.1 \rightarrow 158.0$ & 20 & $489.1 \rightarrow 140.9$ & 56 & 110 \\
\hline 100 & Fluopyram & $\mathrm{F}$ & Approved & Yes & LC & 8.18 & 0.5 & Positive & $397.0 \rightarrow 173.0$ & 40 & $397.0 \rightarrow 145.0$ & 50 & 150 \\
\hline 101 & Fluquinconazole & $\mathrm{F}$ & Approved & Yes & LC & 8.21 & 2.5 & Positive & $376.0 \rightarrow 307.1$ & 56 & $376.0 \rightarrow 108.0$ & 24 & 140 \\
\hline 102 & Flusilazole & F, MB, WP & Not approved & Yes & LC & 8.59 & 1.0 & Positive & $316.1 \rightarrow 247.1$ & 20 & $316.1 \rightarrow 165.0$ & 15 & 160 \\
\hline 103 & Flutolanil & F, MB, WP & Approved & No & LC & 7.87 & 0.5 & Positive & $324.1 \rightarrow 262.1$ & 16 & $324.1 \rightarrow 242.1$ & 24 & 130 \\
\hline 104 & Flutriafol & $\mathrm{F}$ & Approved & Yes & LC & 6.77 & 1.0 & Positive & $302.1 \rightarrow 70.1$ & 16 & $302.1 \rightarrow 122.9$ & 28 & 90 \\
\hline 105 & Fluvalinate tau & I, A & Approved & Yes & LC & 10.81 & 2.5 & Positive & $503.0 \rightarrow 208.0$ & 10 & $503.0 \rightarrow 181.0$ & 26 & 50 \\
\hline 106 & Fonofos & I & Not approved & No & GC & 8.18 & 0.5 & Positive & $246.0 \rightarrow 109.0$ & 5 & $246.0 \rightarrow 137.0$ & 15 & 70 \\
\hline
\end{tabular}




\begin{tabular}{|c|c|c|c|c|c|c|c|c|c|c|c|c|c|}
\hline 107 & Fosthiazate & $\mathrm{AH}, \mathrm{V}$ & Approved & Yes & LC & 6.46 & 0.5 & Positive & $284.0 \rightarrow 104.0$ & 20 & $284.0 \rightarrow 227.8$ & 8 & 110 \\
\hline 108 & Hexaconazole & F, MB, WP & Not approved & Yes & LC & 9.06 & 2.5 & Positive & $316.1 \rightarrow 70.1$ & 20 & $314.1 \rightarrow 70.1$ & 20 & 95 \\
\hline 109 & Hexaflumuron & I & Not approved & No & LC & 9.52 & 5.0 & Negative & $459.1 \rightarrow 439.0$ & 18 & $459.1 \rightarrow 276.1$ & 8 & 100 \\
\hline 110 & Hexythiazox & A & Approved & Yes & LC & 10.10 & 0.5 & Positive & $353.1 \rightarrow 227.9$ & 8 & $353.1 \rightarrow 168.1$ & 24 & 120 \\
\hline 111 & Imidacloprid & I & Approved & Yes & LC & 4.07 & 2.5 & Positive & $256.0 \rightarrow 208.9$ & 12 & $256.0 \rightarrow 175.0$ & 12 & 110 \\
\hline 112 & Indoxacarb & I & Approved & Yes & LC & 9.42 & 1.0 & Positive & $528.1 \rightarrow 293.1$ & 48 & $528.1 \rightarrow 202.8$ & 10 & 140 \\
\hline 113 & Iprodione & $\mathrm{F}, \mathrm{MB}, \mathrm{WP}$ & Not approved & Yes & GC & 13.63 & 10.0 & Positive & $314 \rightarrow 56.0$ & 20 & $314.0 \rightarrow 245.0$ & 10 & 70 \\
\hline 114 & Iprovalicarb & F & Approved & Yes & LC & 8.17 & 0.5 & Positive & $321.2 \rightarrow 119.0$ & 15 & $321.2 \rightarrow 202.9$ & 0 & 108 \\
\hline 115 & Isocarbophos & I & Not approved & Yes & GC & 10.34 & 5.0 & Positive & $230.0 \rightarrow 155.0$ & 25 & $230.0 \rightarrow 198.0$ & 10 & 70 \\
\hline 116 & Isofenphos methyl & I & Not approved & No & LC & 8.75 & 0.5 & Positive & $332.1 \rightarrow 230.9$ & 44 & $332.1 \rightarrow 120.9$ & 10 & 100 \\
\hline 117 & Isoprothiolane & F, MB, WP & Not approved & Yes & LC & 7.87 & 0.5 & Positive & $291.1 \rightarrow 231.1$ & 30 & $291.1 \rightarrow 189.0$ & 12 & 100 \\
\hline 118 & Kresoxim methyl & F & Approved & Yes & LC & 8.74 & 1.0 & Positive & $314.1 \rightarrow 116.0$ & 24 & $314.1 \rightarrow 223.0$ & 15 & 98 \\
\hline 119 & Linuron & $\mathrm{F}$ & Approved & Yes & LC & 7.48 & 1.0 & Positive & $249.0 \rightarrow 160.1$ & 20 & $249.0 \rightarrow 182.3$ & 8 & 120 \\
\hline 120 & Lufenuron & I & Not approved & Yes & $\mathrm{LC}$ & 10.00 & 2.5 & Negative & $509.0 \rightarrow 339.0$ & 5 & $509.0 \rightarrow 326.1$ & 15 & 90 \\
\hline 121 & Malaoxon & I & Not approved & No & LC & 5.99 & 0.5 & Positive & $315.1 \rightarrow 127.2$ & 12 & $315.1 \rightarrow 99.1$ & 12 & 120 \\
\hline 122 & Malathion & I & Not approved & Yes & $\mathrm{LC}$ & 7.87 & 0.5 & Positive & $348.0 \rightarrow 126.7$ & 15 & $348.0 \rightarrow 285.0$ & 8 & 100 \\
\hline 123 & Mandipropamid & $\mathrm{F}$ & Approved & Yes & LC & 7.85 & 0.5 & Positive & $412.1 \rightarrow 328.0$ & 8 & $412.1 \rightarrow 356.1$ & 4 & 130 \\
\hline 124 & Mefenoxam (metalaxyl-M) & $\mathrm{F}$ & Approved & Yes & LC & 6.90 & 0.5 & Positive & $280.0 \rightarrow 220.0$ & 10 & $280.0 \rightarrow 192.0$ & 15 & 110 \\
\hline 125 & Mepanipyrim & F, MB, WP & Approved & Yes & LC & 8.14 & 1.0 & Positive & $224.1 \rightarrow 106.0$ & 30 & $224.1 \rightarrow 77.0$ & 25 & 120 \\
\hline 126 & Metaflumizone & I & Approved & No & LC & 9.87 & 0.5 & Negative & $505.0 \rightarrow 302.0$ & 10 & $541.0 \rightarrow 302.0$ & 20 & 110 \\
\hline 127 & Metalaxyl & $\mathrm{F}$ & Approved & Yes & GC & 9.25 & 0.5 & Positive & $234.0 \rightarrow 146.0$ & 20 & $249.0 \rightarrow 146.0$ & 5 & 70 \\
\hline 128 & Metaldehyde & M & Approved & No & $\mathrm{LC}$ & 3.89 & 20.0 & Positive & $194.1 \rightarrow 61.9$ & 5 & $194.1 \rightarrow 106.0$ & 5 & 50 \\
\hline 129 & Metconazole & $\mathrm{F}$ & Approved & No & $\mathrm{LC}$ & 9.11 & 0.5 & Positive & $320.1 \rightarrow 70.1$ & 33 & $322.1 \rightarrow 70.1$ & 24 & 110 \\
\hline 130 & Methamidophos & I, A & Not approved & Yes & LC & 1.26 & 0.5 & Positive & $142.0 \rightarrow 94.0$ & 12 & $142.0 \rightarrow 125.0$ & 12 & 85 \\
\hline 131 & Methidathion & I, A & Not approved & Yes & LC & 7.05 & 0.5 & Positive & $320.1 \rightarrow 144.8$ & 8 & $320.1 \rightarrow 85.0$ & 30 & 82 \\
\hline 132 & Methiocarb & I, A, M & Not approved & Yes & LC & 7.62 & 0.5 & Positive & $226.1 \rightarrow 169.0$ & 12 & $226.1 \rightarrow 121.1$ & 4 & 90 \\
\hline 133 & Methiocarb sulfone & Met & - & Yes & LC & 4.56 & 1.0 & Positive & $258.1 \rightarrow 122.1$ & 22 & $258.1 \rightarrow 201.1$ & 8 & 100 \\
\hline 134 & Methiocarb sulfoxide & Met & - & Yes & LC & 4.24 & 0.5 & Positive & $242.0 \rightarrow 122.0$ & 28 & $242.0 \rightarrow 185.0$ & 22 & 90 \\
\hline 135 & Methomyl & $\mathrm{I}, \mathrm{A}, \mathrm{AH}$ & Nor approved & Yes & $\mathrm{LC}$ & 3.44 & 1.0 & Positive & $163.1 \rightarrow 88.0$ & 8 & $163.1 \rightarrow 106.0$ & 5 & 80 \\
\hline 136 & Methomyl oxime & Met & - & Yes & $\mathrm{LC}$ & 2.43 & 20.0 & Positive & $106.2 \rightarrow 58.1$ & 10 & $106.2 \rightarrow 42.2$ & 40 & 70 \\
\hline 137 & Methoxyfenozide & I & Approved & Yes & LC & 7.94 & 0.5 & Positive & $369.2 \rightarrow 149.0$ & 10 & $369.2 \rightarrow 313.1$ & 0 & 80 \\
\hline 138 & Metrafenone & $\mathrm{F}$ & Approved & Yes & LC & 9.20 & 2.5 & Positive & $409.1 \rightarrow 209.1$ & 8 & $411.2 \rightarrow 209.1$ & 12 & 120 \\
\hline 139 & Mevinphos (phosdrin) (two isomers) & $\mathrm{I}, \mathrm{A}$ & Not approved & No & LC & $4.35 / 4.85$ & 0.5 & Positive & $225.0 \rightarrow 193.1$ & 0 & $225.0 \rightarrow 127.0$ & 12 & 65 \\
\hline 140 & Monocrotophos & I & Not approved & Yes & LC & 3.69 & 0.5 & Positive & $224.1 \rightarrow 126.8$ & 15 & $224.1 \rightarrow 98.1$ & 12 & 100 \\
\hline 141 & Myclobutanil & F, MB, WP & Approved & Yes & LC & 8.05 & 2.5 & Positive & $289.1 \rightarrow 70.1$ & 16 & $289.1 \rightarrow 125.1$ & 32 & 110 \\
\hline 142 & $\begin{array}{l}\mathrm{N} \cdot N \text {-Dimethyl-N'-p-tolylsulphamide (DMST, } \\
\text { metabolite of tolylfluanid) }\end{array}$ & Met & - & No & LC & 6.01 & 0.5 & Positive & $215.1 \rightarrow 106.1$ & 4 & $215.1 \rightarrow 151.1$ & 10 & 90 \\
\hline 143 & $\begin{array}{l}\mathrm{N} \cdot \mathrm{N} \text {-dimethylformamidine (DMF, metabolite of } \\
\text { amitraz) }\end{array}$ & Met $^{g}$ & - & No & LC & 5.40 & 20.0 & Positive & $149.9 \rightarrow 105.8$ & 30 & $149.9 \rightarrow 122.9$ & 15 & 100 \\
\hline 144 & Nuarimol & F, MB, WP & Approved & No & LC & 7.57 & 2.5 & Positive & $315.0 \rightarrow 252.0$ & 30 & $315.0 \rightarrow 81.1$ & 28 & 80 \\
\hline 145 & Ofurace & F, MB, WP & Approved & No & $\mathrm{LC}$ & 5.94 & 0.5 & Positive & $282.0 \rightarrow 159.9$ & 20 & $282.0 \rightarrow 147.9$ & 30 & 100 \\
\hline 146 & Omethoate & I, A & Not approved & Yes & LC & 2.56 & 0.5 & Positive & $214.1 \rightarrow 124.8$ & 22 & $214.1 \rightarrow 183.0$ & 5 & 84 \\
\hline 147 & Oxadixyl & F, MB, WP & Not approved & Yes & $\mathrm{LC}$ & 5.41 & 0.5 & Positive & $279.1 \rightarrow 219.2$ & 5 & $279.1 \rightarrow 132.3$ & 32 & 110 \\
\hline 148 & Oxamyl & $\mathrm{I}, \mathrm{A}, \mathrm{AH}$ & Approved & Yes & LC & 3.24 & 0.5 & Positive & $237.1 \rightarrow 72.0$ & 12 & $237.1 \rightarrow 90.0$ & 5 & 70 \\
\hline 149 & Oxamyl oxime & Met & - & Yes & LC & 2.76 & 0.5 & Positive & $163.3 \rightarrow 72.1$ & 15 & $163.3 \rightarrow 90.0$ & 10 & 70 \\
\hline 150 & Oxyfluorfen & $\mathrm{H}$ & Approved & No & GC & 11.64 & 5.0 & Positive & $252.0 \rightarrow 146.0$ & 20 & $252.0 \rightarrow 196.0$ & 40 & 70 \\
\hline 151 & Paclobutrazol & $\mathrm{H}$ & Approved & Yes & LC & 7.86 & 1.0 & Positive & $294.1 \rightarrow 70.1$ & 16 & $294.1 \rightarrow 125.2$ & 36 & 115 \\
\hline 152 & Paraoxon methyl & I & Not approved & No & GC & 8.94 & 5.0 & Positive & $230.0 \rightarrow 106.0$ & 20 & $230.0 \rightarrow 136.0$ & 5 & 70 \\
\hline 153 & Parathion ethyl & I & Not approved & No & GC & 9.90 & 5.0 & Positive & $290.9 \rightarrow 109.0$ & 30 & $138.9 \rightarrow 109.0$ & 5 & 70 \\
\hline 154 & Parathion methyl & I & Not approved & Yes & GC & 9.09 & 5.0 & Positive & $263.0 \rightarrow 109.0$ & 15 & $263.0 \rightarrow 79.0$ & 30 & 70 \\
\hline 155 & Penconazole & F, MB, WP & Approved & Yes & LC & 8.80 & 0.5 & Positive & $284.1 \rightarrow 70.1$ & 30 & $284.1 \rightarrow 159.0$ & 15 & 70 \\
\hline 156 & Pencycuron & F, MB, WP & Approved & Yes & LC & 9.26 & 0.5 & Positive & $329.1 \rightarrow 125.1$ & 24 & $329.1 \rightarrow 217.9$ & 12 & 160 \\
\hline 157 & Pendimethalin & $\mathrm{H}$ & Approved & Yes & LC & 10.13 & 2.5 & Positive & $282.2 \rightarrow 212.2$ & 10 & $282.2 \rightarrow 194.1$ & 17 & 80 \\
\hline 158 & Permethrin (two isomers) & I, A & Not approved & Yes & GC & $15.54 / 16.67$ & 5.0 & Positive & $183.1 \rightarrow 168.1$ & 15 & $183.1 \rightarrow 165.1$ & 10 & 70 \\
\hline 159 & Phosalone & I, A & Not approved & No & $\mathrm{LC}$ & 9.13 & 0.5 & Positive & $385.1 \rightarrow 182.0$ & 20 & $385.1 \rightarrow 110.9$ & 55 & 80 \\
\hline 160 & Phosmet & I, A & Approved & Yes & LC & 7.27 & 0.5 & Positive & $318.0 \rightarrow 159.9$ & 16 & $318.0 \rightarrow 133.0$ & 40 & 90 \\
\hline 161 & Phosmet oxon & Met & - & Yes & $\mathrm{LC}$ & 5.32 & 0.5 & Positive & $302.0 \rightarrow 160.0$ & 10 & $302.0 \rightarrow 77.0$ & 55 & 60 \\
\hline 162 & Phthalimide (metabolite folpet) & Met $^{\text {h }}$ & - & No & GC & 5.81 & 5.0 & Positive & $104.0 \rightarrow 50.0$ & 25 & $147.0 \rightarrow 76.0$ & 25 & 70 \\
\hline
\end{tabular}




\begin{tabular}{|c|c|c|c|c|c|c|c|c|c|c|c|c|c|}
\hline \multirow[t]{2}{*}{$\mathrm{N}^{\circ}$} & \multirow[t]{2}{*}{ Compound } & \multirow[t]{2}{*}{ Category $^{a}$} & \multirow{2}{*}{$\begin{array}{l}\text { Legal status in } \\
\text { the } \mathrm{EU}^{\mathrm{b}}\end{array}$} & \multirow{2}{*}{$\begin{array}{l}\text { Subjected to } \\
\mathrm{MRL}^{\mathrm{c}}\end{array}$} & \multirow[t]{2}{*}{ Technique $^{\mathrm{d}}$} & \multirow[t]{2}{*}{ Retention time (min) } & \multirow{2}{*}{$\begin{array}{c}\mathrm{LOQ} \\
\mathrm{ng} \mathrm{g}^{-1}\end{array}$} & \multirow[t]{2}{*}{ Polarity } & \multicolumn{2}{|c|}{ Quantification } & \multicolumn{2}{|c|}{ Confirmation } & \multirow[t]{2}{*}{ Fragmentor } \\
\hline & & & & & & & & & $\begin{array}{l}\text { MRM transition } \\
(m / z)\end{array}$ & $\begin{array}{l}\mathrm{CE} \\
(\mathrm{eV})\end{array}$ & $\begin{array}{l}\text { MRM transition } \\
(\mathrm{m} / \mathrm{z})\end{array}$ & $\begin{array}{l}\mathrm{CE} \\
(\mathrm{eV})\end{array}$ & \\
\hline 163 & Pirimicarb & I & Approved & Yes & LC & 5.17 & 0.5 & Positive & $239.1 \rightarrow 72.1$ & 20 & $239.1 \rightarrow 182.1$ & 12 & 100 \\
\hline 164 & Pirimiphos ethyl & I, A & Not approved & No & LC & 9.86 & 0.5 & Positive & $334.1 \rightarrow 182.1$ & 23 & $334.1 \rightarrow 198.1$ & 25 & 100 \\
\hline 165 & Pirimiphos methyl & I, A & Approved & Yes & LC & 9.08 & 0.5 & Positive & $306.1 \rightarrow 108.1$ & 32 & $306.1 \rightarrow 164.0$ & 20 & 100 \\
\hline 166 & Prochloraz & F, MB, WP & Approved & No & LC & 9.03 & 0.5 & Positive & $376.0 \rightarrow 308.0$ & 10 & $376.0 \rightarrow 70.1$ & 20 & 100 \\
\hline 167 & Procymidone & F, MB, WP & Not approved & Yes & GC & 10.77 & 5.0 & Positive & $283.0 \rightarrow 67.0$ & 40 & $283.0 \rightarrow 68.0$ & 25 & 70 \\
\hline 168 & Profenofos & I, A & Not approved & Yes & LC & 9.67 & 0.5 & Positive & $375.0 \rightarrow 304.8$ & 20 & $373.0 \rightarrow 302.8$ & 20 & 100 \\
\hline 169 & Propargite & A & Not approved & Yes & LC & 10.29 & 0.5 & Positive & $368.2 \rightarrow 231.1$ & 4 & $368.2 \rightarrow 175.0$ & 12 & 88 \\
\hline 170 & Propiconazole & A & Not approved & Yes & $\mathrm{LC}$ & 8.96 & 2.5 & Positive & $342.0 \rightarrow 69.0$ & 21 & $342.0 \rightarrow 159.0$ & 39 & 90 \\
\hline 171 & Propoxur & I & Not approved & No & LC & 5.81 & 0.5 & Positive & $210.1 \rightarrow 111.0$ & 12 & $210.1 \rightarrow 168.1$ & 0 & 70 \\
\hline 172 & Propyzamide (pronamide) & $\mathrm{H}$ & Approved & Yes & LC & 7.86 & 1.0 & Positive & $256.1 \rightarrow 190.0$ & 16 & $256.1 \rightarrow 173.0$ & 25 & 90 \\
\hline 173 & Proquinazid & $\mathrm{F}$ & Approved & Yes & LC & 10.53 & 1.0 & Positive & $372.9 \rightarrow 331.0$ & 20 & $372.9 \rightarrow 289.0$ & 5 & 100 \\
\hline 174 & Prothioconazole-desthio & Met & - & No & LC & 8.44 & 1.0 & Positive & $312.0 \rightarrow 70.1$ & 22 & $312.0 \rightarrow 125.0$ & 18 & 100 \\
\hline 175 & Prothiophos & $\mathrm{F}$ & Not approved & No & GC & 11.43 & 5.0 & Positive & $162.0 \rightarrow 63.1$ & 5 & $266.9 \rightarrow 221.0$ & 20 & 70 \\
\hline 176 & Pyraclostrobin & $\mathrm{F}$ & Approved & Yes & LC & 9.07 & 0.5 & Positive & $388.1 \rightarrow 193.8$ & 8 & $388.1 \rightarrow 163.1$ & 28 & 120 \\
\hline 177 & Pyrazophos & F, MB, WP & Not approved & No & LC & 9.15 & 1.0 & Positive & $374.1 \rightarrow 222.1$ & 23 & $374.1 \rightarrow 194.0$ & 32 & 100 \\
\hline 178 & Pyridaben & I, A & Approved & Yes & LC & 10.68 & 0.5 & Positive & $365.2 \rightarrow 309.0$ & 8 & $309.1 \rightarrow 147.0$ & 16 & 96 \\
\hline 179 & Pyridaphenthion & $\mathrm{I}, \mathrm{A}$ & Not approved & No & LC & 8.06 & 1.0 & Positive & $341.0 \rightarrow 189.0$ & 22 & $341.0 \rightarrow 92.0$ & 34 & 100 \\
\hline 180 & Pyrimethanil & $\mathrm{F}$ & Approved & Yes & GC & 8.22 & 0.5 & Positive & $198.0 \rightarrow 118.0$ & 40 & $198.0 \rightarrow 158.0$ & 20 & 70 \\
\hline 181 & Pyriproxifen & I & Approved & Yes & LC & 10.01 & 0.5 & Positive & $322.2 \rightarrow 96.0$ & 12 & $322.2 \rightarrow 184.9$ & 24 & 80 \\
\hline 182 & Quinalphos & I, A & Not approved & No & LC & 8.67 & 1.0 & Positive & $299.1 \rightarrow 96.9$ & 20 & $299.1 \rightarrow 147.1$ & 30 & 130 \\
\hline 183 & Quinoxyfen & $\mathrm{F}$ & Not approved & Yes & LC & 10.05 & 0.5 & Positive & $308.0 \rightarrow 197.0$ & 32 & $308.0 \rightarrow 161.8$ & 55 & 100 \\
\hline 184 & Rotenone & $\mathrm{I}, \mathrm{R}$ & Not approved & No & LC & 8.58 & 1.0 & Positive & $395.1 \rightarrow 192.1$ & 25 & $395.1 \rightarrow 213.1$ & 20 & 150 \\
\hline 185 & Simazine & I & Not approved & No & LC & 5.79 & 0.5 & Positive & $202.4 \rightarrow 131.9$ & 20 & $202.4 \rightarrow 68.1$ & 30 & 120 \\
\hline 186 & Spirodiclofen & A & Approved & Yes & LC & 10.44 & 0.5 & Positive & $411.1 \rightarrow 71.2$ & 5 & $411.1 \rightarrow 313.0$ & 15 & 110 \\
\hline 187 & Spiromesifen & I & Approved & Yes & LC & 10.21 & 1.0 & Positive & $371.0 \rightarrow 273.0$ & 25 & $273.0 \rightarrow 187.0$ & 15 & 90 \\
\hline 188 & Spirotetramat & I & Approved & No & $\mathrm{LC}$ & 8.26 & 1.0 & Positive & $374.2 \rightarrow 302.2$ & 12 & $374.2 \rightarrow 216.1$ & 36 & 150 \\
\hline 189 & Spirotetramat-enol & Met & - & No & LC & 8.27 & 5.0 & Positive & $302.0 \rightarrow 216.0$ & 20 & $302.0 \rightarrow 270.0$ & 30 & 180 \\
\hline 190 & Spiroxamine (two isomers) & $\mathrm{F}$ & Approved & Yes & GC & $9.02 / 9.47$ & 2.5 & Positive & $100.0 \rightarrow 72.0$ & 5 & $100.0 \rightarrow 58.0$ & 10 & 70 \\
\hline 191 & Tebuconazole & I, A & Approved & Yes & LC & 8.85 & 2.5 & Positive & $308.2 \rightarrow 70.2$ & 22 & $308.2 \rightarrow 125.1$ & 53 & 120 \\
\hline 192 & Tebufenocide & I & Approved & Yes & LC & 8.60 & 0.5 & Positive & $353.1 \rightarrow 132.9$ & 22 & $353.1 \rightarrow 297.1$ & 0 & 98 \\
\hline 193 & Tebufenpyrad & A & Approved & Yes & LC & 9.82 & 0.5 & Positive & $334.2 \rightarrow 117.0$ & 47 & $334.2 \rightarrow 145.0$ & 37 & 180 \\
\hline 194 & Teflubenzuron & I & Not approved & Yes & GC & 5.33 & 0.5 & Positive & $197.0 \rightarrow 135.0$ & 25 & $197.0 \rightarrow 142.0$ & 25 & 70 \\
\hline 195 & Tefluthrin & I & Approved & Yes & GC & 8.34 & 2.5 & Positive & $177.0 \rightarrow 127.0$ & 15 & $177.0 \rightarrow 87.0$ & 35 & 70 \\
\hline 196 & Telodrin (isobenzan) & I & Not approved & No & GC & 10.10 & 2.5 & Positive & $310.8 \rightarrow 274.8$ & 5 & $310.8 \rightarrow 240.8$ & 25 & 70 \\
\hline 197 & Terbufos & $\mathrm{I}, \mathrm{AH}$ & Not approved & No & GC & 8.09 & 2.5 & Positive & $231.0 \rightarrow 129.0$ & 10 & $231.0 \rightarrow 97.0$ & 20 & 70 \\
\hline 198 & Terbuthylazine & $\mathrm{H}$ & Approved & Yes & LC & 7.65 & 0.5 & Positive & $230.0 \rightarrow 174.0$ & 16 & $230.0 \rightarrow 96.0$ & 28 & 100 \\
\hline 199 & Tetrachlorvinphos & I & Not approved & No & LC & 8.67 & 1.0 & Positive & $367.0 \rightarrow 127.0$ & 16 & $364.9 \rightarrow 127.0$ & 16 & 110 \\
\hline 200 & Tetraconazole & $\mathrm{F}, \mathrm{H}$ & Approved & Yes & LC & 8.36 & 5.0 & Positive & $372.0 \rightarrow 159.0$ & 30 & $372.0 \rightarrow 70.1$ & 20 & 100 \\
\hline 201 & Tetradifon & A & Not approved & No & GC & 14.34 & 2.5 & Positive & $158.9 \rightarrow 111.0$ & 15 & $229.0 \rightarrow 201.0$ & 20 & 70 \\
\hline 202 & Tetramethrin & I & Not approved & No & GC & 13.80 & 5.0 & Positive & $164.0 \rightarrow 77.0$ & 30 & $164.0 \rightarrow 107.0$ & 15 & 70 \\
\hline 203 & Thiacloprid & I & Approved & No & LC & 4.76 & 0.5 & Positive & $253.0 \rightarrow 126.0$ & 40 & $253.0 \rightarrow 90.0$ & 16 & 140 \\
\hline 204 & Thiamethoxam & I & Not approved & Yes & LC & 3.56 & 1.0 & Positive & $292.0 \rightarrow 211.1$ & 8 & $292.0 \rightarrow 132.0$ & 22 & 80 \\
\hline 205 & Thiodicarb & I & Not approved & Yes & LC & 6.45 & 0.5 & Positive & $355.1 \rightarrow 88.1$ & 8 & $355.1 \rightarrow 108.1$ & 8 & 60 \\
\hline 206 & Tolclofos methyl & F, MB, WP & Approved & Yes & GC & 9.15 & 0.5 & Positive & $265.0 \rightarrow 93.0$ & 30 & $265.0 \rightarrow 220.0$ & 25 & 70 \\
\hline 207 & Tolylfluanid & $\mathrm{F}$ & No approved & No & GC & 10.56 & 2.5 & Positive & $238.0 \rightarrow 137.0$ & 35 & $238.0 \rightarrow 91.0$ & 35 & 70 \\
\hline 208 & Triadimefon & F, MB, WP & Not approved & Yes & LC & 7.97 & 0.5 & Positive & $294.1 \rightarrow 69.3$ & 20 & $294.1 \rightarrow 197.2$ & 15 & 100 \\
\hline 209 & Triadimenol & F, MB, WP & Not approved & Yes & LC & 8.18 & 2.5 & Positive & $296.1 \rightarrow 70.0$ & 10 & $298.1 \rightarrow 70.0$ & 10 & 80 \\
\hline 210 & Triazophos (hostathion) & I, A & Not approved & Yes & LC & 8.12 & 0.5 & Positive & $314.1 \rightarrow 162.0$ & 19 & $314.1 \rightarrow 118.9$ & 35 & 100 \\
\hline 211 & Trichlorfon & $\mathrm{I}, \mathrm{AH}, \mathrm{V}$ & Not approved & No & LC & 4.29 & 1.0 & Positive & $256.9 \rightarrow 109.0$ & 12 & $258.9 \rightarrow 109.0$ & 12 & 170 \\
\hline 212 & Trifloxystrobin & $\mathrm{F}$ & Approved & Yes & LC & 9.44 & 0.5 & Positive & $409.1 \rightarrow 186.0$ & 12 & $409.1 \rightarrow 145.0$ & 52 & 110 \\
\hline 213 & Triflumizole & $\mathrm{F}$ & Approved & No & LC & 9.49 & 0.5 & Positive & $346.1 \rightarrow 278.0$ & 15 & $346.1 \rightarrow 73.0$ & 4 & 80 \\
\hline 214 & Triflumuron & I & Approved & Yes & LC & 9.13 & 0.5 & Positive & $359.0 \rightarrow 156.0$ & 32 & $359.0 \rightarrow 139.0$ & 8 & 120 \\
\hline 215 & Trifluralin & $\mathrm{H}$ & Not approved & No & GC & 7.17 & 0.5 & Positive & $306.0 \rightarrow 264.0$ & 5 & $264.0 \rightarrow 160.0$ & 15 & 70 \\
\hline 216 & Triticonazole & $\mathrm{F}$ & Approved & No & LC & 8.34 & 2.5 & Positive & $318.1 \rightarrow 70.1$ & 33 & $320.1 \rightarrow 70.1$ & 16 & 110 \\
\hline
\end{tabular}




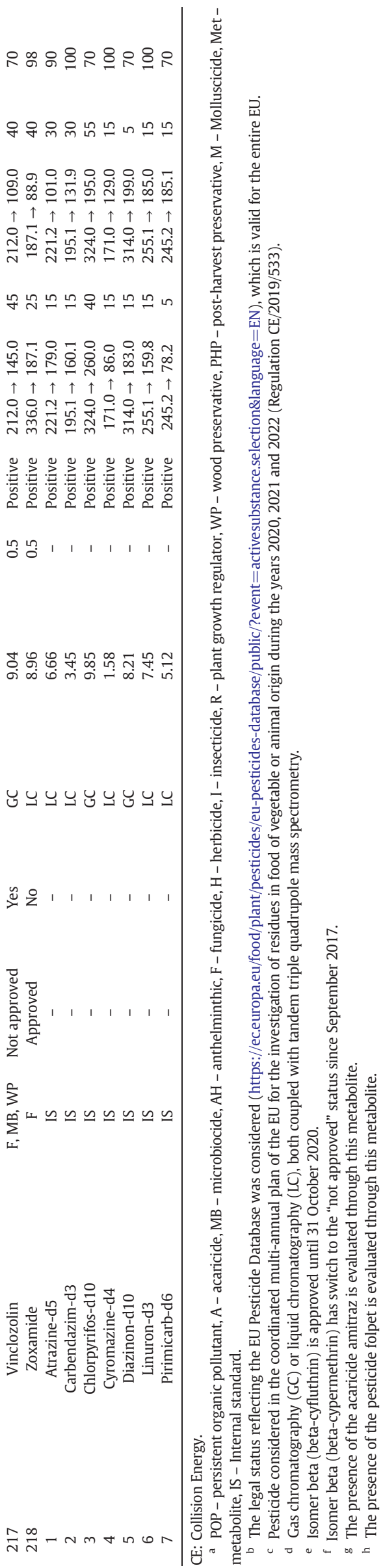

compared with a curve of sucrose solutions with increasing carbon concentration.

\subsection{Sample preparation}

Into a $50 \mathrm{~mL}$ centrifuge tube $10 \pm 0.05 \mathrm{~g}$ of dried and sieved soil plus $10 \mathrm{~mL}$ of ACN-2.5\% FA were added and shaken vigorously. Next, $6 \mathrm{~g}$ of $\mathrm{MgSO}_{4}$ and $1.5 \mathrm{~g}$ of $\mathrm{CH}_{3} \mathrm{COONa}$ were added, shaken vigorously for $1 \mathrm{~min}$ and sonicated for $15 \mathrm{~min}$ (ultrasonic bath, $50 / 60 \mathrm{~Hz}, 120 \mathrm{~W}$ ). Samples were then placed in a rotatory shaker for $25 \mathrm{~min}$. After that, they were centrifuged for $10 \mathrm{~min}$ at $4200 \mathrm{rpm}(3175.16 \times \mathrm{g})$ in a 5804 R Eppendorf centrifuge (Eppendorf, Hamburg, Germany). An aliquot of supernatant extract was filtered through $0.20 \mu \mathrm{m}$ Chromafil@ PET filters (Macherey-Nagel, Düren, Germany). Finally, the supernatant was directly analyzed in GC-MS/MS or diluted with $\mathrm{H}_{2} \mathrm{O}(1: 1, v / \mathrm{v})$ and analyzed in LC-MS/MS.

The samples for recovery experiments and Quality Controls (QCs) were spiked with the required volume to achieve the desired concentration of the standard mix solutions and were left to stand for $1 \mathrm{~h}$ prior to extraction. $100 \mu \mathrm{L}$ of P-IS mix solution was added to all samples in the same step, including the blanks.

\subsection{Instrumental analysis}

\subsubsection{LC-MS/MS}

LC-MSMS analysis of 167 compounds was conducted using a 1290 Infinity II LC System coupled to a Triple Quad 6460 mass spectrometer (Agilent Technologies, Palo Alto, CA, USA). The column was a Poroshell 120 EC-C18 column $(2.1 \times 100 \mathrm{~mm}, 2.7 \mu \mathrm{m}$; Agilent Technologies $)$ equipped with a guard pre-filter with a $0.3 \mu \mathrm{m}$ SS frit and a precolumn $(2.1 \times 5 \mathrm{~mm}, 1.8 \mu \mathrm{m}$; Agilent Technologies $)$ at $50{ }^{\circ} \mathrm{C}$. The mobile phases consisted on $2 \mathrm{mM}$ ammonium acetate $0.1 \% \mathrm{FA}$ in ultrapure water (A) and $2 \mathrm{mM} \mathrm{0.1 \%} \mathrm{FA} \mathrm{ammonium} \mathrm{acetate} \mathrm{in} \mathrm{MEOH}$ (B). A binary gradient using mobile phases $A$ and $B$ was programmed as follows: $5 \% \mathrm{~B}$ - 0.5 min; 5\% B - 1 min; 40\% B - 2.5 min; 85\% B - 8 min; 100\% B - 10 to $14 \mathrm{~min} ; 5 \% \mathrm{~B}-14.01 \mathrm{~min}$ (total run $=18 \mathrm{~min}$ ). The flow rate was $0.4 \mathrm{~mL} \mathrm{~min}^{-1}$ and the injection volume was $5 \mu \mathrm{L}$.

MS/MS analyses were performed using the Agilent Jet Stream Electrospray Ionization Source (AJS-ESI), in both positive and negative ionization mode, with dynamic multiple reaction monitoring (dMRM). The nitrogen supplied by Zefiro 40 nitrogen generator (F-DGSi, Evry, France) was used as desolvation and drying gas. Nitrogen (99.9999\% purity) was used as collision gas. The sheath gas temperature was $330{ }^{\circ} \mathrm{C}$ and the flow rate was $12 \mathrm{~L} \mathrm{~min}^{-1}$. The desolvation and nebulizing gas temperature was $190{ }^{\circ} \mathrm{C}$ and the flow rate was $11 \mathrm{~L} \mathrm{~min}^{-1}$ with a pressure of 26 psi. The capillary voltages were set at 3900 and $2600 \mathrm{~V}$ in positive and negative ionization mode, respectively. The cycle time was $700 \mathrm{~ms}$ and dwell time 3-83 ms.

\subsubsection{GC-MS/MS}

The GC-MS/MS analysis of 51 compounds was performed with a GC System 7890B equipped with a 7693 Autosampler and Triple Quad 7010 mass spectrometer (Agilent Technologies, Palo Alto, USA). The columns were two fused silica ultra-inert capillary columns Agilent J\&WHP-5MS (Crosslinked 5\% phenyl-methyl-polysiloxane, Agilent Technologies) $15 \mathrm{~m}$ length, $0.25 \mathrm{~mm}$ i.d., and $0.25 \mu \mathrm{m}$ film thickness of $0.25 \mu \mathrm{m}$ each connected in series by a Purged Ultimate Union (PUU; Agilent Technologies). The use of two $15 \mathrm{~m}$ columns instead of one of $30 \mathrm{~m}$ permitted the use of the back-flushing technique. Helium (99.999\%) was used as the carrier gas and the flow was adjusted by the retention time lock feature using chlorpyrifos methyl as a reference (retention time $=$ $9.143 \mathrm{~min}$ ). The column temperature was maintained at $80{ }^{\circ} \mathrm{C}$ for $1.8 \mathrm{~min}$, increased to $170{ }^{\circ} \mathrm{C}$ at a rate of $40{ }^{\circ} \mathrm{C} \mathrm{min}{ }^{-1}$, then increased to $310^{\circ} \mathrm{C}$ at a rate of $10^{\circ} \mathrm{C} \mathrm{min}-1$ and held for $3 \mathrm{~min}$. The injection volume was $1.6 \mu \mathrm{L}$ in splitless mode using a $4 \mathrm{~mm}$ Ultra Inert Liner with glass wool (Agilent Technologies) and it was set at $250{ }^{\circ} \mathrm{C}$. Each 
chromatographic analysis lasted $20.75 \mathrm{~min}$. Post-run backflush was set at $-5.8 \mathrm{~mL} \mathrm{~min}^{-1}$ and $315^{\circ} \mathrm{C}$ for $5 \mathrm{~min}$.

MS/MS analyses were performed using electron impact (EI) ionization source in multiple reaction monitoring (MRM) mode. The EI source temperature was set at $280{ }^{\circ} \mathrm{C}$. Nitrogen 6.0 (99,9999\% purity, Linde, Dublin, Ireland) was used as the collision gas at a flow of $1.5 \mathrm{~mL} \mathrm{~min}^{-1}$. The transfer line temperature was $280^{\circ} \mathrm{C}$. A solvent delay of $3.7 \mathrm{~min}$ was left. The cycle time was in the range of 52-334 ms and the dwell time was between 15 and $40 \mathrm{~ms}$.

\subsection{Method validation parameters}

The validation of the developed method was performed following the recommendations of the European Union SANTE 12682/2019 and the SANCO 825/00 Rev.1 guidance document on residue analytical methods (EC, 2010; EC, 2019b), which were followed in the absence of specific guidelines for the analysis of pesticide residues of pesticides in soil. The procedures are detailed in the accompanying Data in Brief article entitled "Supporting dataset on the optimization of a QuEChERSbased method for the determination of 218 pesticide residues in clay loam soil".

\section{Results and discussion}

\subsection{Optimization of chromatographic and spectrometric conditions}

\subsubsection{LC-MS/MS}

The optimization of MS/MS conditions for each compound, including the search for the precursor and product ions and the collision energy, was performed injecting directly to the mass spectrometer $5 \mu \mathrm{L}$ of either individual solutions or discrete mixtures of maximum 15 compounds at $1 \mu \mathrm{g} \mathrm{mL}-1 /$ each in ACN. The mobile phases used in this stage were $2 \mathrm{mM}$ ammonium formate $0.1 \%$ AA in ultrapure water (A) and in $\mathrm{MEOH}(\mathrm{B})$ in isocratic mode (50:50, $v / v)$. The precursor ion and the fragmentor were selected using a MS2 scan. The product ions were optimized using product ion scanning at different collision energies, choosing those with the higher response.

Most of the analytes were determined in positive mode except for a few of which gave a better response in negative mode (Table 1). The precursor ions corresponded to $[\mathrm{M}+\mathrm{H}]^{+}$and $[\mathrm{M}+\mathrm{H}]^{-}$in positive and negative mode, respectively, except for some of them (acrinathrin, aldicarb, cyhalothrin (lambda), etofenprox, famoxadone, fenpropathrin, malathion, metaldehyde, methidathion, oxamyl, phosalone and propargite) which formed ammonium adducts $[\mathrm{M}+\mathrm{NH} 4]^{+}$. For other analytes (abamectin, aldicarb sulfone, dinocap, fenbutatin oxide and flubendiamide), specific ions were used as precursor ions. In some compounds containing elements with a characteristic isotope distribution in their structure, such as $\mathrm{Cl}$ and $\mathrm{Br}$ in hexaconazole and metrafenone, respectively, transitions corresponding to ${ }^{35} \mathrm{Cl}$ and ${ }^{37} \mathrm{Cl}$ or ${ }^{79} \mathrm{Br}$ and ${ }^{81} \mathrm{Br}$ were selected. The transitions were selected in terms of selectivity and sensitivity, choosing the most abundant for quantification proposes.

After selecting the best combination of MRM transitions for each target analyte and P-IS, chromatographic and source conditions were optimized. These experiments were performed by injecting standards at $20 \mathrm{ng} \mathrm{mL}{ }^{-1}$ in ACN (in triplicate). The final conditions selected were then tested with a matrix-matched standard at the same concentration.

The source parameters were optimized using Mass Hunter Source Optimizer software (Agilent Technologies, Palo Alto, USA). These parameters include gas temperature, gas flow, nebulizer gas pressure, sheath gas flow and temperature, capillary voltage and nozzle voltage.

Two reverse phase columns were tested for the chromatographic separation performance: the ZORBAXEclipse Plus C18 $(2.1 \mathrm{~mm} \times 50 \mathrm{~mm}$, $1.8 \mu \mathrm{m}$, Agilent Technologies) and the Poroshell 120 EC-C18 column $(2.1 \times 100 \mathrm{~mm}, 2.7 \mu \mathrm{m}$; Agilent Technologies). The Poroshell column showed a better performance in terms of peak shape and column pressures (under 400 bar while with the ZORBAX column was over 600 bar), which is better for long-term equipment protection. The mobile phase and its gradient were optimized to obtain a good separation of the compounds along the chromatogram. The phase modifiers ammonium acetate, ammonium formate, FA and AA were compared. No major differences were achieved with any of the weak acids and $0.1 \%$ FA was selected after choosing it as solvent extraction modifier (see Section 3.2 Optimization of the Extraction Method). On the other hand, better areas and peak shapes were obtained when using $2 \mathrm{mM}$ ammonium acetate in water and organic phase solvent.

To study the injection volume we tested 1, 2, 5, 10 and $20 \mu \mathrm{L}$, being $5 \mu \mathrm{L}$ the final choice due to the good responses obtained. The use of higher volumes resulted in detector saturation. Finally, the dilution of the sample extract was assessed. The selected compounds belong to different chemical groups and, therefore, have a wide range of polarities which could result in poor chromatographic separations. The dilution of the final extract, in ACN-2.5\% FA, not only contributed to reduce possible matrix effects, but also produced better peak shapes for compounds such as monocrotophos. The selected sample dilution was water-extract $1: 1(\mathrm{v} / \mathrm{v})$ as a compromise between sensitivity, peak shape and selectivity for all compounds.

\subsubsection{GC-MS/MS}

Our laboratory had an important database of compound transitions for GC-MS/MS which were optimized for the needs of the method. Firstly, all available transitions for the target analytes were tested by injecting a $100 \mathrm{ng} \mathrm{mL}^{-1}$ mix standard solution in order to find out the most promising transitions. Due to the complexity of the soil matrix, transitions were selected giving priority to selectivity over sensitivity to avoid possible interference from the matrix components. This was evaluated by comparing $100 \mathrm{ng} \mathrm{mL}^{-1}$ of the prepared mix standard solution in the solvent against that of the matrix extract. Once the best transitions were selected, the optimal collision energies were determined by injecting the standard solution in a range of $0-65 \mathrm{~V}$, tested in $5 \mathrm{~V}$ increments. The transitions were distributed in 24 MRM windows to achieve good sensitivity, dwells, and cycle time.

As mentioned throughout the text, ACN-2.5\% FA extracts were injected directly into the gas chromatographer, although this is not the most common solvent chosen for GC analyses. This decision was taken after noting that some analytes were lost or partially degraded during the evaporation steps required for a solvent change. In addition, ACN has proven to be a suitable solvent for GC injection in other method development papers (Mastovska and Lehotay, 2004). Adequate peak shape and sensitivity were achieved even at low concentrations for all analytes.

The chromatographic parameters had been previously optimized by our group, so no further modifications were made either to the oven temperature ramp or to the column type due to the good results obtained for similar analytes (Luzardo et al., 2015; Luzardo et al., 2014). However, as the solvent chosen in those methods had been cyclohexane, it was necessary to optimize some parameters. The initial oven temperature was set at $80^{\circ} \mathrm{C}$, close to the boiling point of $\mathrm{ACN}$, to avoid condensation in the column. The injector temperature and the injection volume were studied in the range of $230-290{ }^{\circ} \mathrm{C}$ and $1-1.6 \mu \mathrm{L}$, respectively, taking into account the vapor pressure solvent in the liner. Finally, the temperature of the ionization source and the temperature of the transfer line were studied in increments of $10{ }^{\circ} \mathrm{C}$ from 250 to $320{ }^{\circ} \mathrm{C}$ and $270-320{ }^{\circ} \mathrm{C}$, respectively.

The precursor and product ions, retention times, fragmentation and collision energies of all the compounds are shown in Table 1. Fig. 1 shows the chromatograms of a blank soil sample spiked at $20 \mathrm{ng}$ g1 with the target analytes and P-IS by the LC-MS/MS and GC-MS/MS analyses. 


\subsection{Optimization of the extraction method}

A QuEChERS-based method for the extraction of target pesticides in the soil was investigated. Some parameters, such as the combination of salts, the acidity of the extraction solvent, moisture of the sample and some mechanical changes in the procedure were studied in order to achieve the best extraction efficiency. To facilitate the optimization, all experiments were done at the same concentration of $20 \mathrm{ng} \mathrm{g}^{-1}$ in triplicate.

We decided not to modify the sample quantity used in the original QuEChERS procedure ( $10 \mathrm{~g}$ ) as it provides a combination of practicality and representativeness. Similarly, ACN was used as the extraction solvent because of its suitability for a wide range of compound polarities and no higher volume $(10 \mathrm{~mL})$ was required as sufficient supernatant volume was collected. Our first approach was to introduce a few modifications in the mechanical aspects of the procedure. A 15-min sonication step from a time-course experiment (up to two hours) was chosen. This step was included just after adding the QuEChERS salts and vigorously shaking the tubes, to produce further decomposition of possible aggregates (Asensio-Ramos et al., 2010). Likewise, 25 min of rotatory shaking were introduced after the sonication step to increase the interface and contact time between the extractant solvent and the soil.

The two main official variants of the original QuEChERS, the AOAC method (Lehotay, 2007) and the EN method (EN, 2019), were compared in order to select the most appropriate for the target pesticides and metabolites and the soil matrix: $1.5 \mathrm{~g}$ of $\mathrm{NaOAc}$ and $6 \mathrm{~g} \mathrm{MgSO}_{4}$, in the $\mathrm{AOAC}$ procedure, and $4 \mathrm{~g} \mathrm{MgSO}_{4}, 1 \mathrm{~g} \mathrm{NaCl}, 1 \mathrm{~g}$ sodium citrate dihydrate and $0.5 \mathrm{~g}$ sodium hydrogen citrate sesquihydrate in the EN method. After centrifugation, the extract was either collected for analytical determination or further purified. The clean-up is intended to reduce matrix components that may remain in the extract after the first extraction step. This step is typically achieved by d-SPE using secondary amine (PSA), C18 and graphitized carbon black (GCB), either alone or in mixtures (Pszczolinska and Michel, 2016). Nevertheless, we have seen that these sorbents usually retain some of the more polar compounds reducing recoveries, which is consistent with what other authors have described (Caldas et al., 2011). Instead, the d-SPE clean-up was approached by the use of Enhance Matrix Removal (EMR), a proprietary lipid removal sorbent patented by Agilent Technologies (Agilent, 2015), that had not been tested in soil samples previously. The mean recoveries obtained in these experiments (AOAC vs. EN QuEChERS extraction, with or without an additional EMR-clean-up step) are graphically presented in Fig. 1 of the accompanying Data in Brief. In general, the recovery percentages and peak shapes for most of the 218 compounds included in this method were better with the AOAC salts. Not surprisingly, the clean-up did not provide any improvement but on the contrary, a large amount of the compounds either reduced their extraction performance or were lost. Therefore, the addition of a clean-up step (either with PSA, C18, GCB, or EMR) was discarded because it hardly improved selectivity and appeared to have minor changes of the matrix effect (Supplementary Figs. 1-3).

The original AOAC method for the analysis of plants uses $1 \% \mathrm{AA}$ in the extraction solvent to adjust the $\mathrm{pH}$. Nevertheless, we identified the percentage and type of acid employed to acidify the ACN as the next variable to be optimized in adapting this method to the pesticide extraction from clay loam soils. Therefore, in addition to AA, we also tested FA and compared the extraction in the presence of $1 \%$ of each acid with that of no acid added. It was noted that acidification definitely improved the recovery for the vast majority of analytes. In addition, we also verified that this improvement was higher with FA instead of with AA. Then, to find out the optimized percentage of FA, it was tested at $0.5 \%, 1 \%$, and $2.5 \%$. We did not consider the inclusion of higher percentages of acid, since it has been described that extreme $\mathrm{pH}$ conditions can affect the stability of many pesticides (i.e. acetamiprid is unstable at pHs below 4 and above 7) (Schilder, 2008). From our results, it can be concluded that the extraction efficiency of most of the chemicals under study improved with the increase of FA in the solvent. Thus, ACN acidified with 2.5\% F.A was set as the extraction solvent. The detailed results are shown in Figs. 2 and 3 of the accompanying Data in Brief article.

The original QuEChERS method was developed for fruits and vegetables, which are samples with high water content (> 70\%). Therefore, when processing dry samples with QuEChERS it is common to add some amount of water (Grande-Martinez et al., 2015), and in fact, in soil samples it has been described that the addition of water could make the pores more accessible to the extraction solvent (Pinto et al., 2010). Thus, adding water to the soil samples was the next step in the optimization process. Deionized water was added to aliquots of the air-dried soil sample to reach 10, 20, 30, 40 and 50\% of moisture. The samples were left to hydrate for one hour. We did not observe large differences in the recoveries of the compounds determined in LC/MS-MS technique with the increase of moisture. Nonetheless, as the percentage of moisture increased, the results were worse for many of the compounds analyzed by GC-MS/MS. A possible explanation could be the remaining water in the extract which not only is not a suitable solvent for GC chromatography but also reduced the matrix load in the sample and, therefore, the sensibility. To solve this problem of excess polarity of the injection solvent in the GC-MS/MS (ACN containing a certain percentage of water) it would have been necessary to add an evaporation step of the obtained extract to completely eliminate the water and be able to change the solvent, which had been previously discarded (detailed results in Fig. 4, Data in Brief).

\subsection{Method validation}

The optimized QuEChERS-based method was validated in terms of linearity, accuracy and precision following the agreements stated in the "Method Validation Parameters" section.

The complexity of the soil matrix may have some effect on the analysis, either by suppressing or enhancing the response, that may compromise the accuracy, selectivity and sensitivity of the method (Asensio-Ramos et al., 2010; Matuszewski et al., 2003). In order to assess these possible interferences in the chromatographic response, a matrix effect study was performed. The calibration curves, either in the matrix extract or in the solvent (both diluted with water $(1: 1, v / v)$ for LC-MS/MS) were prepared in the range of $6.25-50 \mathrm{ng} \mathrm{mL}^{-1}$ in triplicate. The matrix effect (ME) was evaluated comparing the response obtained for each analyte in the soil extract with that given in the solvent at the same concentration in both equipment, extracting the blank to the soil matrix signal. No significant matrix effects were considered when ME was between 80 and 120\% (EC, 2010; EC, 2019b). Fiftyfour compounds presented significant enhancement caused by the co-extraction of matrix components, fifty of which were compounds analyzed by GC-MS/MS, representing $98 \%$ of the total compounds analyzed by this technique. These results have shown the importance of evaluating the matrix effect on this equipment, where the presence of high amounts of matrix components could protect the analyte from adsorption or degradation during evaporation in the inlet (Fernandes et al., 2013). In contrast, the main trend observed for ME in LC-MS/MS was towards signal suppression although this was not significant for most of them ( 135 compounds) and only 6 presented strong or medium signal suppression. However, to improve the accuracy of the quantification, as well as to simplify the procedure, it was decided to use matrixmatched calibration in both techniques (detailed results in Fig. 5, Data in Brief).

The linearity was obtained from a triplicate range of 9 levels (from 0.39 to $100 \mathrm{ng} \mathrm{g}^{-1}$ ). The response was satisfactory for all the compounds, with a deviation of back-calculated concentration from true concentration $<20 \%$, for both GC-MS/MS and LC-MS/MS, either in matrix extract or in matrix-extract diluted with water $(1: 1, \mathrm{v} / \mathrm{v})$, respectively. $R^{2}$ values were above 0.99 for all analytes in both techniques (Table 2, Data in Brief). 

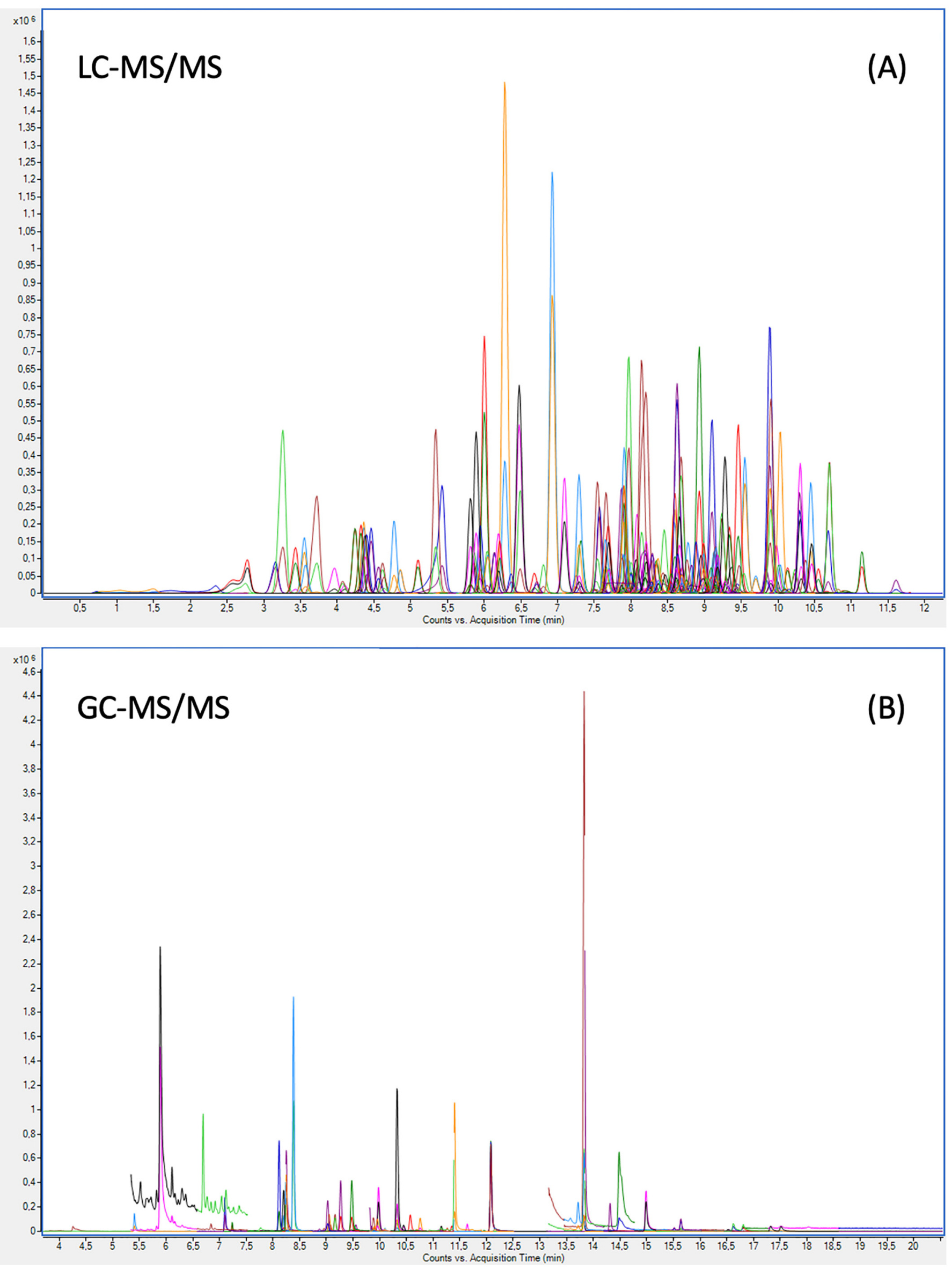


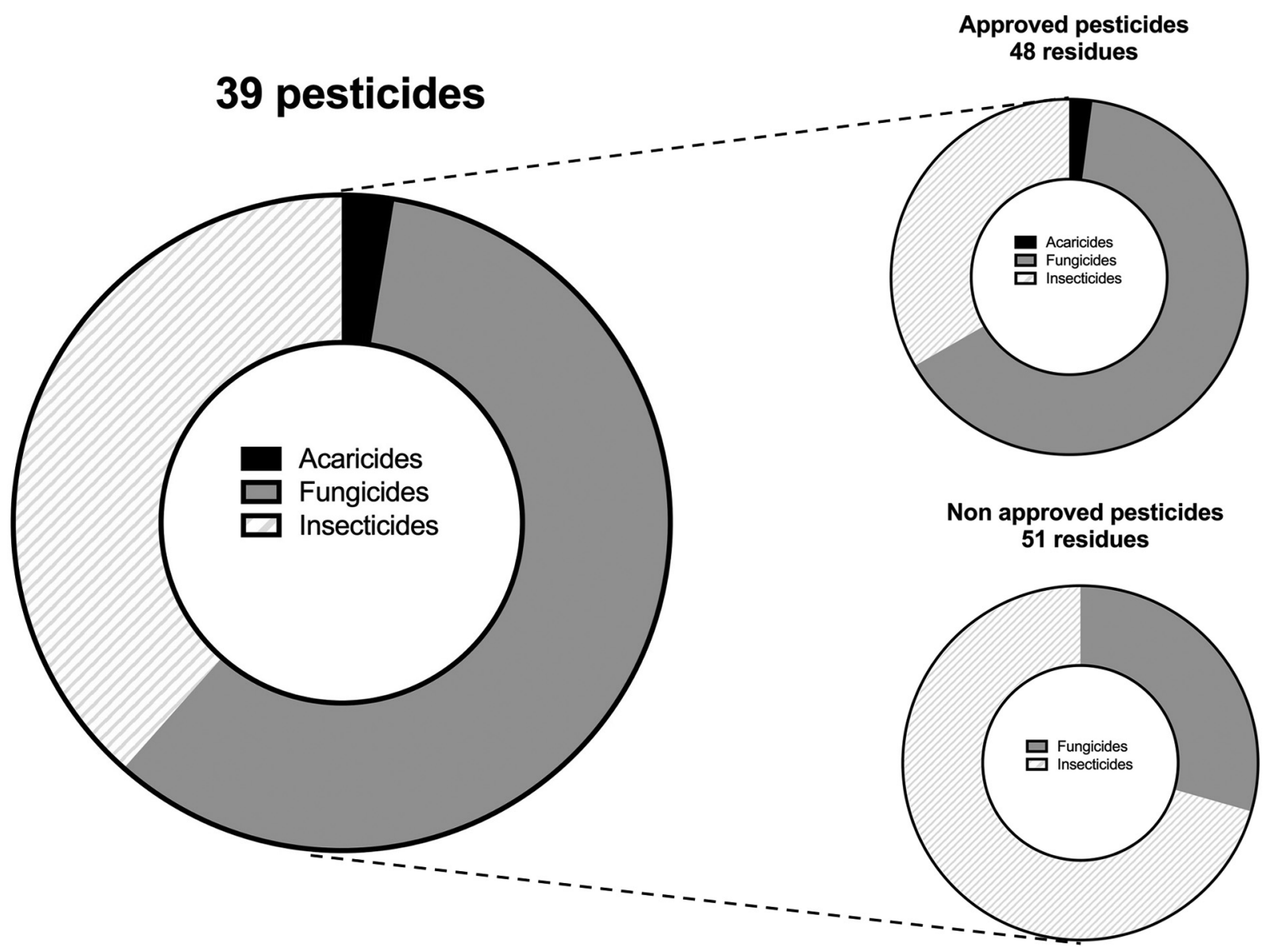

Fig. 2. Distribution of pesticide residues detected in the 18 soil samples, classified by target pest.

The accuracy and the precision of the method were tested with recovery experiments at 7 concentration levels in quintuplicate: $0.5,1.0$, $2.5,5,10,20$ and $50 \mathrm{ng} \mathrm{g}^{-1}$. The highest concentration of this range is usually selected as an acceptable LOQ for pesticide residue analyses in soil (EC, 2010; EC, 2019b). The method presented satisfactory accuracy (recoveries in the range 70-120\%) and precision (RSD < 20\%) for 198 analytes ( $90.8 \%$ of the compounds) for all the fortification levels ranging from the highest concentration $\left(50 \mathrm{ng} \mathrm{g}^{-1}\right)$ to the LOQ set for each analyte. As the LOQ we chose the lowest level of fortification that met all the validation criteria. Although 18 compounds presented recoveries over $120 \%$ or below $70 \%$ between their LOQ and $50 \mathrm{ng} \mathrm{g}^{-1}$, they were included in the final method due to their importance for pesticide monitoring in soils. In fact, the SANTE guidelines take this situation into account while allow for the validation of compounds in the range of $60-140 \%$ if they show high reproducibility (RSD $<20 \%$ ) in routine analyses, which was the case for these compounds (EC, 2019a). Additionally, two compounds (dichloran and nuarimol) presented recoveries between 70 and $120 \%$ at $5 \mathrm{ng} \mathrm{g}^{-1}$, but a high variability (RSD > 20\%). However, this is also acceptable for concentration levels below $10 \mathrm{ng} \mathrm{g}^{-1}$ in pesticide residues in soil, according to the guidelines we have followed (EC, 2010). In total, $90 \%$ of the compounds had an LOQ equal to $5 \mathrm{ng} \mathrm{g}^{-1}$ or below, which is 10 times lower than the typically fixed value for these residues in soil. On the other hand, the Limits of Detection (LODs) were determined as the lowest point of the calibration curve having a signalto-noise ratio above 3 (Peak-to-Peak algorithm) and having acceptable accuracy (80-120\%). For this purpose, matrix-matched calibration curves in the range of 0.024 to $100 \mathrm{ng} \mathrm{g}^{-1}$ were prepared in triplicate and injected in each equipment. Detailed results are presented in Table 2 of the accompanying Data in Brief.

After the validation process, the proposed method proves to be accurate and reliable for the analysis of the pesticides selected in this study in clay loam soil samples.

\subsection{Application of the method to real samples}

The developed method was applied to soil samples from two different scenarios. Firstly, a series of 18 soil samples from farmlands were analyzed, all of them with the characteristics of the soil employed in the method validation. In addition, two soil samples from the investigation on a wildlife poisoning case were also analyzed.

\subsubsection{Monitoring of agricultural land}

The samples came from 3 farm vineyards, 3 mixed vegetable farms, 3 fruit tree farms, 3 banana farms, 3 farms with crop in transition to organic production, 1 avocado farm, and 2 abandoned farmlands. The summary of the results obtained is shown in Table 2. It should be noted that there were only three samples in which no pesticide residues were detected (one abandoned farm, the avocado farm, and one fruit tree farm). Thus, the detection range was 0 to 18 residues per sample, although most (61\%) ranged 2 to 6 residues. A total of 109 residues above LOQ were identified, belonging to 39 different pesticides (and metabolites), which represents $17.9 \%$ of the analytes included in the 


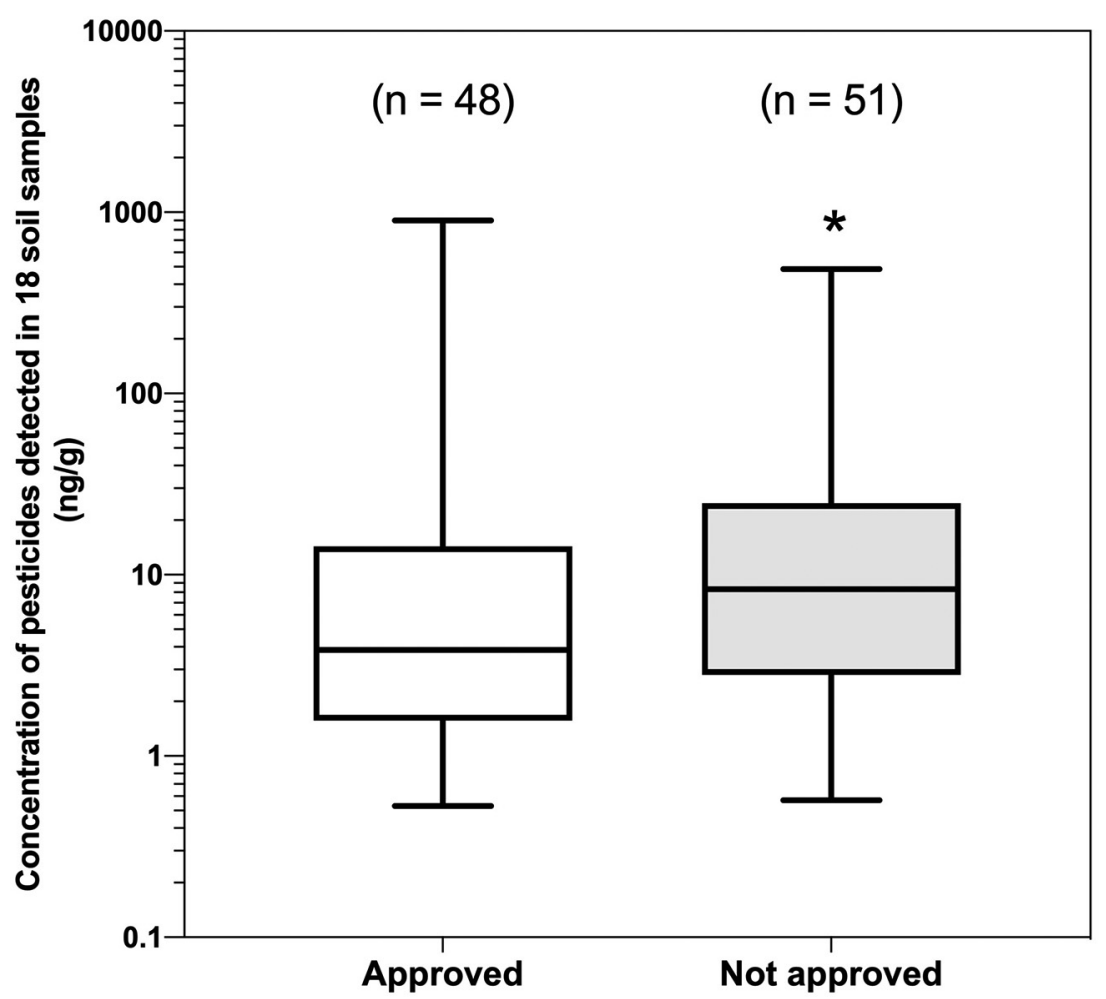

TYPE OF PESTICIDE

\begin{tabular}{|l|l|}
\hline & \\
\hline Mann Whitney test & \\
\hline $\mathrm{P}$ value & 0.0474 \\
\hline Exact or approximate $\mathrm{P}$ value? & Exact \\
\hline $\mathrm{P}$ value summary & ${ }^{\star}$ \\
\hline Significantly different $(\mathrm{P}<0.05) ?$ & Yes \\
\hline One- or two-tailed $\mathrm{P}$ value? & Two-tailed \\
\hline Sum of ranks in column $\mathrm{A}, \mathrm{B}$ & 2117,2833 \\
\hline
\end{tabular}

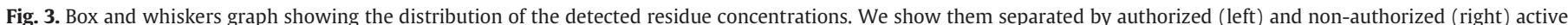

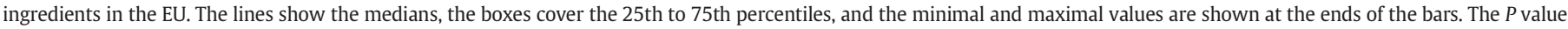
was calculated by the Mann-Whitney $U$ test because the series of values did not fit the normal distribution (Kolgomorov-Smirnoff test).

method. Of these, 9 were detected in more than $20 \%$ of the soil samples $\left(4,4^{\prime}\right.$ dichlorobenzophenone (DBP), fenbutatin oxide, buprofezin, alpha-endosulfan, fenamiphos sulfoxide, benalaxyl, boscalid, fenarimol and penconazole). Of these frequently detected compounds, most are approved for cultivation in the EU (EC, 2020). It is worth noting the case of DBP, since it is the most frequently detected residue $(72 \%$ of the samples), although at relatively low concentrations. Although it is identified in Tables 1-2 as "metabolite of dicofol", the truth is that DBP could also originate (in rare cases) from 4, 4'-DDT by fungal degradation (Xerocomus chrysenteron), or from chlorobenzilate or other organochlorine compounds. The only exception is soil \#9 (Table 2) in which $25 \mathrm{ng} \mathrm{g}^{-1}$ of dicofol was also detected. We believe it is possible to state that the insecticide dicofol was applied on this farm in a relatively recent time. However, in all other cases we cannot assure that the residue comes from the application of dicofol.

We noted that EU non-authorized pesticides were higher both in number of residues (Fig. 2) and in concentration (Fig. 3). The most frequently detected class of pesticide among the authorized active substances was fungicides. Some of them are already known to remain strongly retained in the soil such as fenbutatin oxide (Gray et al., 1995), boscalid (He et al., 2020) or benalaxyl (Qin et al., 2014). For others, such as buprofezin (Oulkar et al., 2009) or penconazole (Abd-Alrahman and Ahmed, 2012), a rapid degradation rate (especially in loamy soils) has been described, and its detection would point to a very recent use of these products. In contrast, most of the detected residues of unauthorized active substances belong to the group of insecticides (Fig. 2).

Fig. 3 shows the distribution of the concentrations found, divided also between unauthorized and authorized substances. As can be seen, although the highest residue values found in soil correspond to some authorized substances (such as deltamethrin in soil \#11, Table 2), the residue concentration values of the unauthorized active substances are significantly higher than those of residues of the authorized active substances.

From our results it cannot be deduced that there is a type of crop that generates a greater amount of residue in the soil, and the variability is high. However, the small sample size of the study precludes any firm conclusions.

The case of soil \#16 called our attention. This sample presented the greatest number of residues $(n=18)$, and comes from a farm vineyard, whose phytosanitary treatment records we know about. 

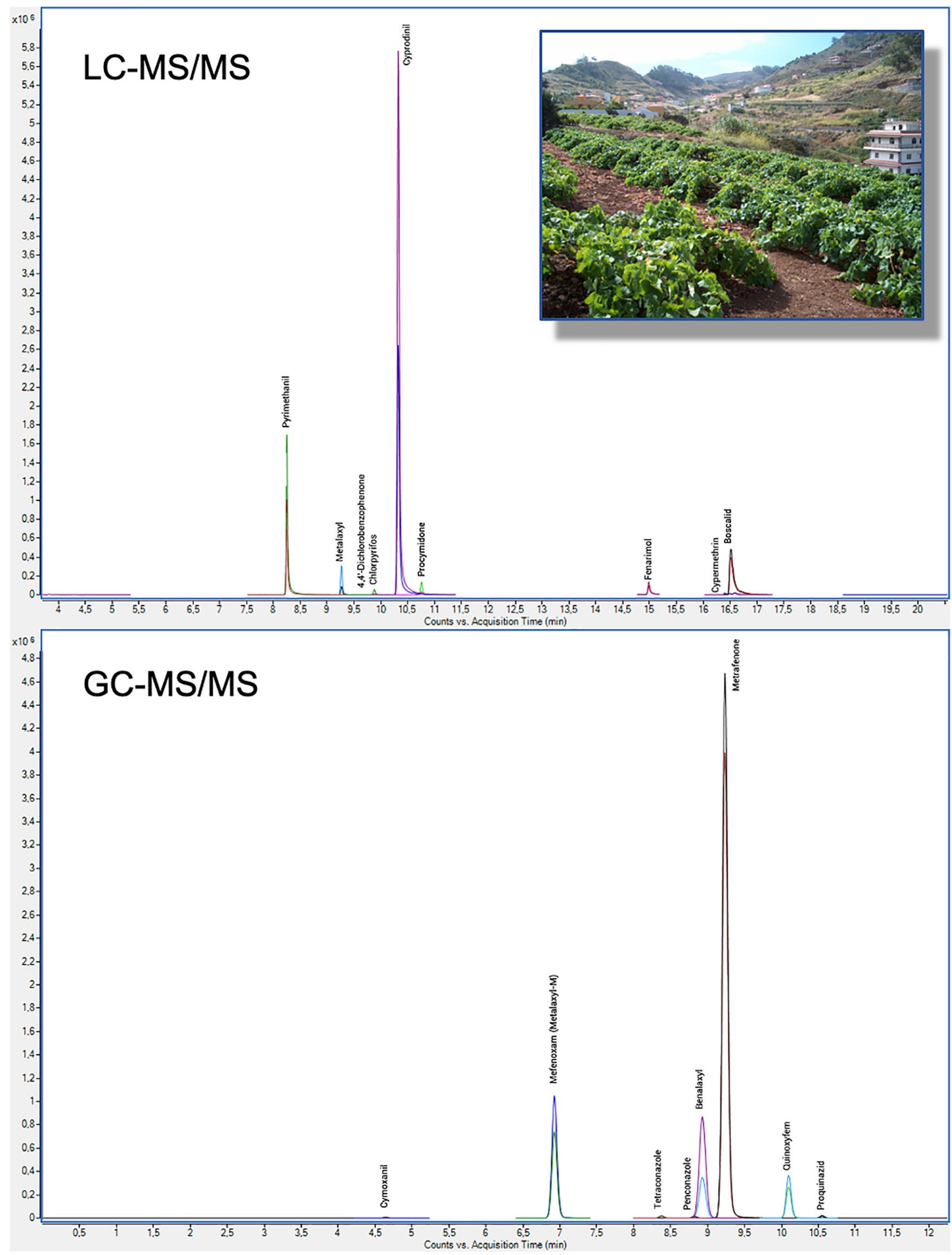

Fig. 4. Chromatograms with the identification of the 18 residues detected in soil sample \#16 (small-scale family-run vineyard). 


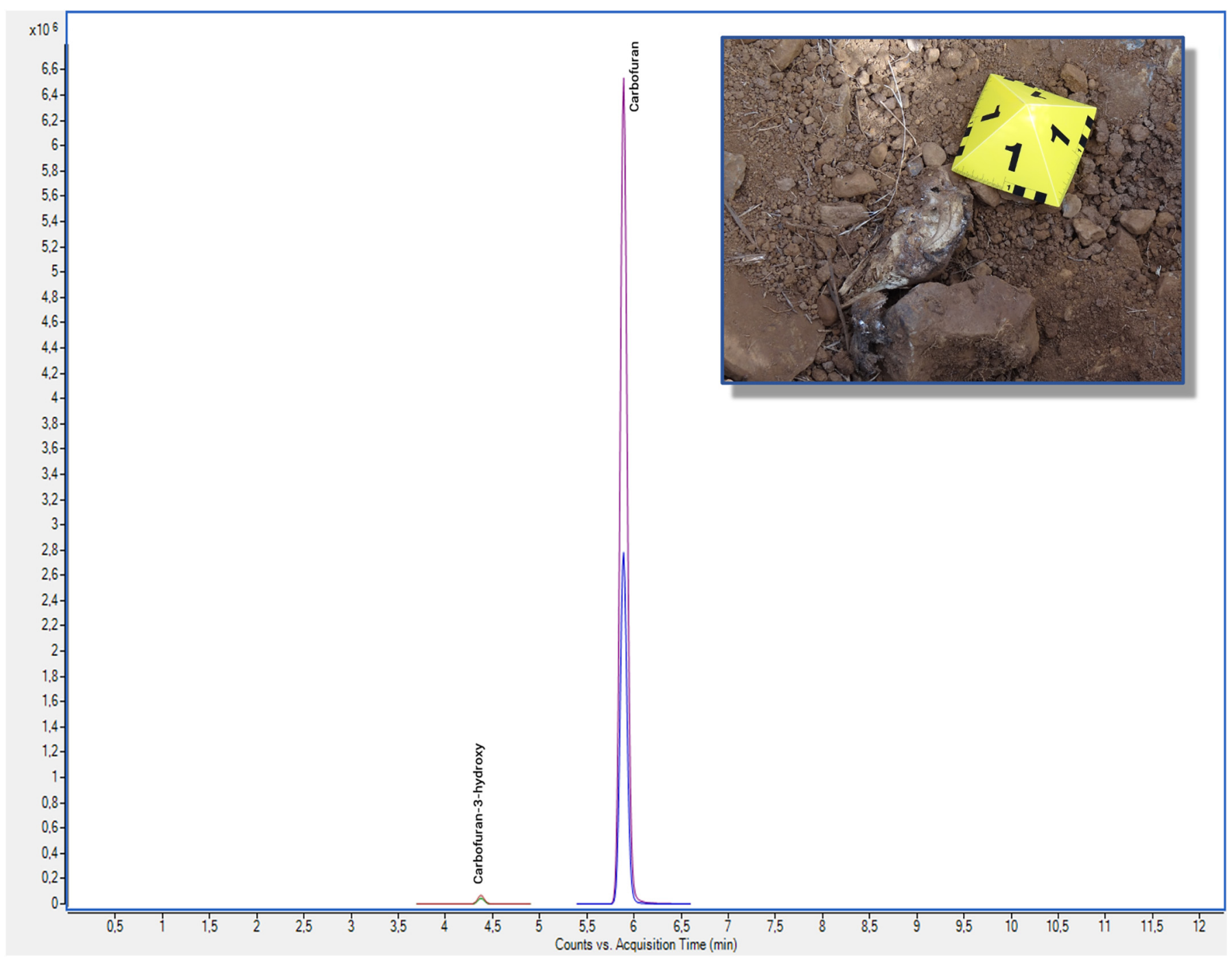

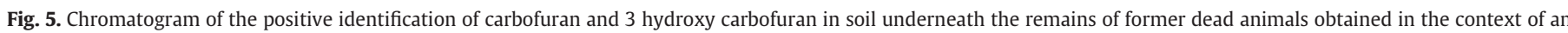
environmental forensic investigation.

In Fig. 4 we show the chromatograms corresponding to the analysis of this sample, as an example of the potential of the method we have developed. The residues that have been detected in this sample faithfully reflect the phytosanitary treatment records applied in this farm during the last 10 years. All the residues detected in the sample correspond to pesticides used in the past on the plot. Of the 18 pesticides identified, the highest concentrations $\left(>100 \mathrm{ng} \mathrm{g}^{-1}\right)$ correspond to pesticides used during the last three seasons, except for cyprodinil (not used on this farm for about 4-5 years) and quinoxyfen, which has been not used for more than 10 years (EC, 2020). Cypermethrin residues correspond to a winter treatment in 2017, since this pesticide has not been applied on the farm since then.

\subsubsection{Environmental Forensic Investigation}

In this case, we applied the method to two soil samples, collected beneath highly degraded carcass remains and bones of birds of prey that had allegedly died from poisoning in the past. The samples were collected by court order, after a large number of bird and fresh domestic animal carcasses were detected in the area, as well as many dead insects. The aim of the environmental toxicology expert's report was to identify whether the former remains also belonged to animals that may had been poisoned in a previous episode. For this, we analyzed the soil beneath the animal remains in our laboratory. As shown in Fig. 5, the application of the method described here allowed the identification of carbofuran, and 3 hydroxy carbofuran at extremely high concentrations (4198 and $227 \mathrm{ng} \mathrm{g}^{-1}$, respectively). Carbofuran was banned in the EU in 2007, but it has been shown it is still often used for malicious purposes, such as in the preparation of poisoned baits (Ruiz-Suarez et al., 2015). This analysis allowed us to confirm that the alleged poisoner probably acted in the area previously with the same modus operandi, since our results on the soil were consistent with the detection of the same compounds in the fresh bird carcasses recently found in the same area.

\section{Conclusions}

A one-step QuEChERS-based method has been developed and validated for the extraction of 218 pesticides in agricultural soil samples and their analysis by LC-MS/MS and GC-MS/MS. This makes the method a simpler, faster and cheaper process as the need to use more solvents and purification reagents were avoided. A successful linearity, precision, accuracy was obtained for the selected compounds and the LOQ was well below the typically fixed in soil. The method was tested on various farms soil samples and forensic cases and 
Table 2

Pesticides and metabolites detected in 18 samples of agricultural soil. Concentrations are expressed in $\mu \mathrm{gg}^{-1}$.

\begin{tabular}{|c|c|c|c|c|c|c|c|c|c|c|c|c|c|c|c|c|c|c|c|}
\hline Soil sample & 1 & 2 & 3 & 4 & 5 & 6 & 7 & 8 & 9 & 10 & 11 & 12 & 13 & 14 & 15 & 16 & 17 & 18 & Frequency \\
\hline Type of crop/farm & $\mathrm{AF}^{\mathrm{a}}$ & $\mathrm{AF}^{\mathrm{a}}$ & $\mathrm{B}^{\mathrm{b}}$ & $\mathrm{B}^{\mathrm{b}}$ & $\mathrm{B}^{\mathrm{b}}$ & $\mathrm{F}^{\mathrm{c}}$ & $\mathrm{F}^{\mathrm{c}}$ & $\mathrm{F}^{\mathrm{c}}$ & $\mathrm{MV}^{\mathrm{d}}$ & $\mathrm{MV}^{\mathrm{d}}$ & $\mathrm{MV}^{\mathrm{d}}$ & $\mathrm{AV}^{\mathrm{e}}$ & $\mathrm{OT}^{\mathrm{f}}$ & $\mathrm{OT}^{\mathrm{f}}$ & $\mathrm{OT}^{\mathrm{f}}$ & $V Y^{g}$ & $V Y^{g}$ & $\mathrm{VY}^{\mathrm{g}}$ & $\%$ \\
\hline $\begin{array}{l}\text { 4.4'-Dichlorobenzophenone } \\
\text { (metabolite of dicofol) }\end{array}$ & & & 4.93 & 4.06 & 2.03 & 4.86 & 14.36 & & 94.72 & 16.29 & 4.37 & & 4.31 & & 3.80 & 1.42 & 0.75 & 0.68 & 72.2 \\
\hline $4.4^{\prime}$-Dicofol & & & & & & & & & 24.84 & & & & & & & & & & 5.6 \\
\hline Benalaxyl & 1.54 & & & & & & & & & & & & & & & 27.05 & 0.53 & 0.62 & 22.2 \\
\hline Boscalid (formely nicobifen) & 5.58 & & & & & & & & & & & & & & & 536.97 & 26.68 & 24.08 & 22.2 \\
\hline Buprofezin & & & 0.58 & 0.55 & 1.20 & 4.06 & 0.56 & & 48.54 & 2.41 & 3.64 & & & & & & & & 44.4 \\
\hline Chlorpyrifos & 4.92 & & & & & & & & & & & & & & & 7.75 & & & 11.1 \\
\hline Cymoxanil & & & & & & & & & & & & & & & & 3.90 & & & 5.6 \\
\hline $\begin{array}{l}\text { Cypermethrin (sum of four } \\
\text { isomers) }\end{array}$ & & & & & & & & & & & & & & & & 108.56 & & & 5.6 \\
\hline $\begin{array}{l}\text { Cyproconazole (two } \\
\text { isomers) }\end{array}$ & & & & & & & & & & & & & & & 2.94 & & & & 5.6 \\
\hline Cyprodinil & & & & & & & & & & & & & & & & 401.24 & & & 5.6 \\
\hline Deltamethrin & & & & & & & & & & & 902.19 & & & & & & & & 5.6 \\
\hline Endosulfan alfa & & & & & & & & & 19.43 & 1.49 & 1.82 & & 51.31 & & 6.74 & & & & 27.8 \\
\hline Ethion (diethion) & & & & & & & & & & & & & & & & 0.57 & & & 5.6 \\
\hline Ethoprophos & & & & & & & & & 0.61 & 0.58 & & & & & & & & & 11.1 \\
\hline Fenamiphos & & & & 6.15 & 42.80 & & & & 2.72 & & 0.58 & & & & & & & & 22.2 \\
\hline Fenamiphos sulfone & & & & & 0.83 & & & & & & 3.81 & & & & & & & & 11.1 \\
\hline Fenamiphos sulfoxide & & & & 2.05 & 6.23 & & & & 1.96 & 1.73 & 4.90 & & & & & & & & 27.8 \\
\hline Fenarimol & & & & & & & & & & & & & & & & 8.78 & & & 5.6 \\
\hline Fenbutatin oxide & & & 4.79 & 12.64 & 8.34 & 46.69 & 14.75 & & 385.27 & 361.86 & 29.13 & & 9.80 & & 9.36 & & & & 55.6 \\
\hline Fenpropimorph & & & & & & & & & & & & & & 6.19 & & & & & 5.6 \\
\hline Hexythiazox & & & & & & & & & 2.95 & & & & & & & & & & 5.6 \\
\hline Imidacloprid & & & & & & & 5.14 & & & & & & & & & & & & 5.6 \\
\hline Lufenuron & 3.39 & & & & & & & & & & & & & & & & & & 5.6 \\
\hline Mefenoxam (metalaxyl-M) & & & & & & & & & & & & & & & & 14.98 & & & 5.6 \\
\hline Metalaxyl & & & & & & & & & & & & & & & & 12.41 & & & 5.6 \\
\hline Methoxyfenozide & & & & & & & & & 1.28 & & & & & & & & & & 5.6 \\
\hline Metrafenone & & & & & & & & & & & & & & & & 488.33 & 11.26 & & 11.1 \\
\hline Myclobutanil & & & & & & & 2.41 & & & & 2.45 & & & & & & & & 11.1 \\
\hline $\mathrm{N} \cdot N$-Dimethyl-N & & & & & & & & & & & & & & & & & & & \\
\hline $\begin{array}{l}\text { '-p-tolylsulphamide } \\
\text { (DMST.metabolite of } \\
\text { tolyfluanid) }\end{array}$ & & & & & & 1.05 & 2.80 & & & & & & & & & & & & 11.1 \\
\hline Oxadixyl & & & & & & & & & & & & & & & 2.09 & & & & 5.6 \\
\hline Penconazole & & & & & & 0.92 & 1.64 & & & & & & & & & 3.90 & & 0.57 & 22.2 \\
\hline Permethrin (two isomers) & 4.17 & & & & & & & & & & & & & & & & & & 5.6 \\
\hline Procymidone & & & & & & & & & & & & & & & & 18.60 & & & 5.6 \\
\hline Proquinazid & & & & & & & & & & & & & & & & 4.80 & & & 5.6 \\
\hline Pyrimethanil & & & & & & & & & & & & & & & & 31.43 & & & 5.6 \\
\hline Quinoxyfen & & & & & & & & & & & & & & & & 390.30 & 11.35 & 6.16 & 16.7 \\
\hline Tetraconazole & & & & & & & & & & & & & & & & 9.64 & & & 5.6 \\
\hline Triadimefon & & & & & & 1.86 & 2.57 & & 0.86 & & & & & & & & & & 16.7 \\
\hline Triadimenol & & & & & & 9.96 & 35.42 & & 2.68 & & & & & & & & & & 16.7 \\
\hline Number of residues & 5 & 0 & 3 & 5 & 6 & 7 & 9 & 0 & 12 & 6 & 9 & 0 & 3 & 1 & 5 & 18 & 5 & 5 & \\
\hline $\begin{array}{l}\text { a } \text { AF: abandoned farmland. } \\
\text { b } \text { B: banana crop. } \\
\text { c F: fruit tree farms. } \\
\text { d } \text { MV: mixed vegetables farms } \\
\text { e } \text { AV: avocado farm; } \\
\text { f } \text { OT: organic transition farms; } \\
\text { g VY: vineyards. }\end{array}$ & & & & & & & & & & & & & & & & & & & \\
\hline
\end{tabular}

demonstrated to be well suited for monitoring pesticide residues in this matrix.

Supplementary data to this article can be found online at https://doi. org/10.1016/j.scitotenv.2020.142015.

\section{CRediT authorship contribution statement}

1. guarantor of integrity of the entire study: OPL

2. study concepts and design: $A A D, O P L$

3. literature research: $A A D, C R B, R D D, M M B S, O P L$

4. laboratory work: $A A D, C R B, R D D, M M B S, O P L$

5. data analysis: $A A D, C R B, O P L$

6. statistical analysis: $A A D, C R B, O P L$

7. manuscript preparation: $A A D, C R B, R D D, M M B S, O P L$

8. manuscript editing: $A A D, C R B, R D D, M M B S, O P L$

\section{Declaration of competing interest}

The authors declare no conflict of interest. This is an independent research. Mention of trade names or commercial products does not constitute endorsement or recommendation for use.

\section{Acknowledgements}

This research was supported by the Spanish Ministry of Education, Culture and Sports via a doctoral grant to the first author Andrea Acosta-Dacal (FPU16-01888) and also supported by the University of Las Palmas de Gran Canaria via a doctoral grant to the co-first author Cristian Rial-Berriel (ULPGC-012-2016). The authors would like to thank Dr. Jesús Notario from the Edaphology Department of the University of La Laguna for his help in the identification of the soil samples as 
clay loam soil, and Mrs. Ana Macías Montes for her technical assistance in the Toxicology Laboratory of the University of Las Palmas de Gran Canaria.

\section{References}

Abd-Alrahman, S.H., Ahmed, N.S., 2012. Dissipation of penconazole in tomatoes and soil. Bull. Environ. Contam. Toxicol. 89, 873-876.

Agilent, 2015. Technical Note-Recommended Protocols for Enhanced Matrix RemovalLipid. Agilent Technologies, Inc, USA, p. 2015.

de Andrea, M.M., Papini, S., Nakagawa, L.E., 2001. Optimizing microwave-assisted solvent extraction (MASE) of pesticides from soil. J. Environ. Sci. Health B 36, 87-93.

Asensio-Ramos, M., Hernandez-Borges, J., Ravelo-Perez, L.M., Rodriguez-Delgado, M.A. 2010. Evaluation of a modified QuEChERS method for the extraction of pesticides from agricultural, ornamental and forestal soils. Anal. Bioanal. Chem. 396, 2307-2319.

Ashworth, J., Keyes, D., Kirk, R., Lessard, R., 2001. Standard procedure in the hydrometer method for particle size analysis. Commun. Soil Sci. Plant Anal. 32, 633-642.

Barron, M.G., Ashurova, Z.J., Kukaniev, M.A., Avloev, H.K., Khaidarov, K.K., Jamshedov, J.N., Rahmatullova, O.S., Atolikshoeva, S.S., Mamadshova, S.S., Manzenyuk, O., 2017. Residues of organochlorine pesticides in surface soil and raw foods from rural areas of the Republic of Tajikistan. Environ. Pollut. 224, 494-502.

Caldas, S.S., Bolzan, C.M., Cerqueira, M.B., Tomasini, D., Furlong, E.B., Fagundes, C., Primel, E.G., 2011. Evaluation of a modified QuEChERS extraction of multiple classes of pesticides from a rice paddy soil by LC-APCI-MS/MS. J. Agric. Food Chem. 59, 11918-11926.

Castro, J., Sanchez-Brunete, C., Tadeo, J.L., 2001. Multiresidue analysis of insecticides in soil by gas chromatography with electron-capture detection and confirmation by gas chromatography-mass spectrometry. J. Chromatogr. A 918, 371-380.

Djurovic, R., Gajic Umiljendic, J., Cupac, S., Ignjatovic, L., 2010. Solid phase microextraction as an efficient method for characterization of the interaction of pesticides with different soil types. J. Braz. Chem. Soc. 21, 985-994.

Djurovic, J., Djordjevic, T., Radivojevic, L., Santric, L., Gajic-Umiljendic, J., 2012. Multiresidue analysis of pesticides in soil by liquid-solid extraction procedure. Pestic i Fitomedicina 27, 239-244.

EC, 2000. Directive 2000/60/EC of the European Parliament and of the council of 23 October 2000 establishing a framework for community action in the field of water policy. Off. J. Eur. Union L 327, 1-73.

EC, 2010. SANCO/825/00 Rev. 8.1. Guidance Document on Pesticide Residue Analytical Methods. 2010. European Commission. Directorate General Health and Consumer Protection. pp. 1-28.

EC, 2019a. Commission implementing regulation (EU) 2019/533 concerning a coordinated multiannual control programme of the union for 2020, 2021 and 2022 to ensure compliance with maximum residue levels of pesticides and to assess the consumer exposure to pesticide residues in and on food of plant and animal origin. Off. J. Eur. Union L88/28.

EC, 2019b. SANTE/12682/2019. Guidance Document on Analytical Quality Control and Method Validation Procedures for Pesticide Residues and Analysis in Food and Feed. 2019b. European Commission. Directorate General for Health and Food Safety.

EC, 2020. EU Pesticides Database. 2020. European Commission.

EN, 2019. European Norm 15662. Foods of Plant Origin - Multimethod for the Determination of Pesticide Residues Using GC- and LC-Based Analysis Following Acetonitrile Extraction/Partitioning and Clean-up by Dispersive SPE-Modular QuEChERS-Method. European Commitee for Standardization.

Eudoxie, G.D., Mathurin, G., Lopez, V., Perminova, O., 2019. Assessment of pesticides in soil from obsolete pesticides stores: a Caribbean case study. Environ. Monit. Assess. $191,498$.

Fenoll, J., Hellin, P., Martinez, C.M., Flores, P., 2009. Multiresidue analysis of pesticides in soil by high-performance liquid chromatography with tandem mass spectrometry. J. AOAC Int. 92, 1566-1575.

Fernandes, V.C., Domingues, V.F., Mateus, N., Delerue-Matos, C., 2013. Multiresidue pesticides analysis in soils using modified QuEChERS with disposable pipette extraction and dispersive solid-phase extraction. J. Sep. Sci. 36, 376-382.

Fernandez-Alvarez, M., Llompart, M., Lamas, J.P., Lores, M., Garcia-Jares, C., Cela, R. Dagnac, T., 2008. Simultaneous determination of traces of pyrethroids, organochlorines and other main plant protection agents in agricultural soils by headspace solid-phase microextraction-gas chromatography. J. Chromatogr. A 1188, 154-163.

Forero-Mendieta, J.R., Castro-Vargas, H.I., Parada-Afonso, F., Guerrero-Dallos, J.A., 2012. Extraction of pesticides from soil using supercritical carbon dioxide added with methanol as co-solvent. J. Supercrit. Fluids 68, 64-70.

Grande-Martinez, A., Arrebola, F.J., Moreno, L.D., Vidal, J.L., Frenich, A.G., 2015. Development and validation of a multiresidue method for the determination of pesticides in dry samples (rice and wheat flour) using liquid chromatography/triple quadrupole tandem mass spectrometry. J. AOAC Int. 98, 1186-1198.

Gray, A., Dutto, A.J., Eadsforth, C.V., 1995. Fenbutatin oxide, fate in soil after extensive commercial use in Italy and Spain. Pestic. Sci. 43, 295-302.

Han, Y., Mo, R., Yuan, X., Zhong, D., Tang, F., Ye, C., Liu, Y., 2017. Pesticide residues in nutplanted soils of China and their relationship between nut/soil. Chemosphere 180, 42-47.

He, Y., Meng, M., Yohannes, W.K., Khan, M., Wang, M., Abd El-Aty, A.M., Hacimuftuoglu, F., He, Y., Gao, L., She, Y., 2020. Dissipation pattern and residual levels of boscalid in cucumber and soil using liquid chromatography-tandem mass spectrometry. J. Environ. Sci. Health B 55, 388-395.
Hwang, J.I., Zimmerman, A.R., Kim, J.E., 2018. Bioconcentration factor-based management of soil pesticide residues: Endosulfan uptake by carrot and potato plants. Sci. Total Environ. 627, 514-522.

Karasali, H., Marousopoulou, A., Machera, K., 2016. Pesticide residue concentration in soil following conventional and Low-Input Crop Management in a Mediterranean agroecosystem, in Central Greece. Sci. Total Environ. 541, 130-142.

Kosubova, P., Skulcova, L., Polakova, S., Hofman, J., Bielska, L., 2020. Spatial and temporal distribution of the currently-used and recently-banned pesticides in arable soils of the Czech Republic. Chemosphere 254, 126902.

Kumari, B., Madan, V.K., Kathpal, T.S., 2008. Status of insecticide contamination of soil and water in Haryana, India. Environ. Monit. Assess. 136, 239-244.

Lehotay, S.J., 2007. Determination of pesticide residues in foods by acetonitrile extraction and partitioning with magnesium sulfate: collaborative study. J. AOAC Int. 90 485-520.

Lesueur, C., Gartner, M., Mentler, A., Fuerhacker, M., 2008. Comparison of four extraction methods for the analysis of 24 pesticides in soil samples with gas chromatographymass spectrometry and liquid chromatography-ion trap-mass spectrometry. Talanta 75, 284-293.

Luzardo, O.P., Ruiz-Suarez, N., Valeron, P.F., Camacho, M., Zumbado, M., HenriquezHernandez, L.A., Boada, L.D., 2014. Methodology for the identification of 117 pesticides commonly involved in the poisoning of wildlife using GC-MS-MS and LC-MSMS. J. Anal. Toxicol. 38 (3), 155-163.

Luzardo, O.P., Almeida-Gonzalez, M., Ruiz-Suarez, N., Zumbado, M., HenriquezHernandez, L.A., Meilan, M.J., Camacho, M., Boada, L.D., 2015. Validated analytica methodology for the simultaneous determination of a wide range of pesticides in human blood using GC-MS/MS and LC-ESI/MS/MS and its application in two poisoning cases. Sci. Justice 55, 307-315.

Masia, A., Vasquez, K., Campo, J., Pico, Y., 2015. Assessment of two extraction methods to determine pesticides in soils, sediments and sludges. Application to the Turia Rive Basin. J. Chromatogr. A 1378, 19-31.

Mastovska, K., Lehotay, S.J., 2004. Evaluation of common organic solvents for gas chromatographic analysis and stability of multiclass pesticide residues. J. Chromatogr. A 1040, 259-272.

Matuszewski, B.K., Constanzer, M.L., Chavez-Eng, C.M., 2003. Strategies for the assessment of matrix effect in quantitative bioanalytical methods based on HPLC-MS/MS. Anal. Chem. 75, 3019-3030.

Merdassa, Y., Liu, J.F., Megersa, N., 2013. Development of a one-step microwave-assisted extraction method for simultaneous determination of organophosphorus pesticides and fungicides in soils by gas chromatography-mass spectrometry. Talanta 114 227-234.

Otalvaro, J.O., Brigante, M., 2018. Interaction of pesticides with natural and synthetic solids. Evaluation in dynamic and equilibrium conditions. Environ. Sci. Pollut. Res. Int. 25, 6707-6719.

Oulkar, D.P., Banerjee, K., Patil, S.H., Upadhyay, A.K., Taware, P.B., Deshmukh, M.B., Adsule, P.G., 2009. Degradation kinetics and safety evaluation of buprofezin residues in grape (Vitis vinifera L.) and three different soils of India. Pest Manag. Sci. 65, 183-188.

Pastor-Belda, M., Garrido, I., Campillo, N., Vinas, P., Hellin, P., Flores, P., Fenoll, J., 2015. Dispersive liquid-liquid microextraction for the determination of new generation pesticides in soils by liquid chromatography and tandem mass spectrometry. J. Chromatogr. A 1394, 1-8.

Perestrelo, R., Silva, P., Porto-Figueira, P., Pereira, J.A.M., Silva, C., Medina, S., Camara, J.S. 2019. QuEChERS-fundamentals, relevant improvements, applications and future trends. Anal. Chim. Acta 1070, 1-28.

Pinto, C.G., Laespada, M.E., Martin, S.H., Ferreira, A.M., Pavon, J.L., Cordero, B.M., 2010. Simplified QuEChERS approach for the extraction of chlorinated compounds from soil samples. Talanta 81, 385-391.

Pszczolinska, K., Michel, M., 2016. The QuEChERS approach for the determination of pesticide residues in soil samples: an overview. J. AOAC Int. 99, 1403-1414.

Qin, F., Gao, Y.X., Guo, B.Y., Xu, P., Li, J.Z., Wang, H.L., 2014. Environmental behavior of benalaxyl and furalaxyl enantiomers in agricultural soils. J. Environ. Sci. Health B 49, 738-746.

Redondo, M.J., Ruiz, M.J., Boluda, R., Font, G., 1996. Optimization of a solid-phase extraction technique for the extraction of pesticides from soil samples. J. Chromatogr. A 719, 69-76.

Ruiz-Suarez, N., Boada, L.D., Henriquez-Hernandez, L.A., Gonzalez-Moreo, F., SuarezPerez, A., Camacho, M. Zumbado, M. Almeida-Gonzalez, M., 2015. Del Ma Travieso-Aja M, Luzardo OP. Continued implication of the banned pesticides carbofuran and aldicarb in the poisoning of domestic and wild animals of the Canary Islands (Spain). Sci. Total Environ. 505, 1093-1099.

Salemi, A., Rasoolzadeh, R., Nejad, M.M., Vosough, M., 2013. Ultrasonic assisted headspace single drop micro-extraction and gas chromatography with nitrogen-phosphorus detector for determination of organophosphorus pesticides in soil. Anal. Chim. Acta 769 121-126.

Schilder, A., 2008. Effect of Water pH on the Stability of Pesticides. 2008. Michigan State University Extension. Michigan State University, USA.

Silva, V., Mol, H.G.J., Zomer, P., Tienstra, M., Ritsema, C.J., Geissen, V., 2019. Pesticide residues in European agricultural soils - a hidden reality unfolded. Sci. Total Environ. 653 1532-1545.

Snyder, J.L., Grob, R.L., Oostdyk, T.S., McNally, M.E., 1994. Supercritical fluid extraction of selected pesticides from fortified soils and determination by gas chromatography with electron capture detection. J. Environ. Sci. Health A 29, 1801-1816.

Suszter, G.K., Ambrus, A., 2017. Distribution of pesticide residues in soil and uncertainty of sampling. J. Environ. Sci. Health B 52, 557-563.

Tor, A., Aydin, M.E., Özcan, S., 2006. Ultrasonic solvent extraction of organochlorine pesticides from soil. Anal. Chim. Acta 559, 173-180. 
Vasickova, J., Hvezdova, M., Kosubova, P., Hofman, J., 2019. Ecological risk assessment of pesticide residues in arable soils of the Czech Republic. Chemosphere 216, 479-487.

Vidal, J.L.M., Padilla Sánchez, J., Plaza Bolaños, P., Frenich, A.G., Romero González, R., 2010 Use of pressurized liquid extraction for the simultaneous analysis of 28 polar and 94 non polar pesticides in agricultural soils by GC-QqQ-MS/MS and UPLC/QqQ-MS/MS J. AOAC Int. 93, 1715-1731.

Wang, R., Yuan, Y., Yen, H., Grieneisen, M., Arnold, J., Wang, D., Wang, C., Zhang, M., 2019 A review of pesticide fate and transport simulation at watershed level using SWAT: current status and research concerns. Sci. Total Environ. 669, 512-526.

Wong, F., Alegria, H.A., Bidleman, T.F., 2010. Organochlorine pesticides in soils of Mexico and the potential for soil-air exchange. Environ. Pollut. 158, 749-755.

Zhang, K., Wong, J.W., Yang, P., Tech, K., Dibenedetto, A.L., Lee, N.S., Hayward, D.G., Makovi, C.M., Krynitsky, A.J., Banerjee, K., Jao, L., Dasgupta, S., Smoker, M.S.
Simonds, R., Schreiber, A., 2011. Multiresidue pesticide analysis of agricultural commodities using acetonitrile salt-out extraction, dispersive solid-phase sample cleanup, and high-performance liquid chromatography-tandem mass spectrometry. J. Agric. Food Chem. 59, 7636-7646.

Zhao, P., Wang, Z., Li, K., Guo, X., Zhao, L., 2018. Multi-residue enantiomeric analysis of 18 chiral pesticides in water, soil and river sediment using magnetic solid-phase extraction based on amino modified multiwalled carbon nanotubes and chiral liquid chromatography coupled with tandem mass spectrometry. J. Chromatogr. A 1568, 8-21.

Zhou, Q., Wang, J., Meng, B., Cheng, J., Lin, G., Chen, J., Zheng, D., Yu, Y., 2013. Distribution and sources of organochlorine pesticides in agricultural soils from central China. Ecotoxicol. Environ. Saf. 93, 163-170. 